\title{
Global Sensitivity Analysis of High Speed Shaft Subsystem of a Wind Turbine Drive Train
}

\author{
Saeed Asadi (iD, Viktor Berbyuk, and Håkan Johansson \\ Department of Mechanics and Maritime Sciences, Chalmers University of Technology, 41296 Göteborg, Sweden \\ Correspondence should be addressed to Saeed Asadi; saeed.asadi@chalmers.se
}

Received 21 September 2017; Revised 21 December 2017; Accepted 25 December 2017; Published 1 February 2018

Academic Editor: Ryoichi Samuel Amano

Copyright (C) 2018 Saeed Asadi et al. This is an open access article distributed under the Creative Commons Attribution License, which permits unrestricted use, distribution, and reproduction in any medium, provided the original work is properly cited.

\begin{abstract}
The wind turbine dynamics are complex and critical area of study for the wind industry. Quantification of the effective factors to wind turbine performance is valuable for making improvements to both power performance and turbine health. In this paper, the global sensitivity analysis of validated mathematical model for high speed shaft drive train test rig has been developed in order to evaluate the contribution of systems input parameters to the specified objective functions. The drive train in this study consists of a 3phase induction motor, flexible shafts, shafts' coupling, bearing housing, and disk with an eccentric mass. The governing equations were derived by using the Lagrangian formalism and were solved numerically by Newmark method. The variance based global sensitivity indices are introduced to evaluate the contribution of input structural parameters correlated to the objective functions. The conclusion from the current research provides informative beneficial data in terms of design and optimization of a drive train setup and also can provide better understanding of wind turbine drive train system dynamics with respect to different structural parameters, ultimately designing more efficient drive trains. Finally, the proposed global sensitivity analysis (GSA) methodology demonstrates the detectability of faults in different components.
\end{abstract}

\section{Introduction}

Nowadays wind turbine industry is growing very fast and wind power has become one of the most cost effective of all renewable energy sources because of environmental concerns and finite resource of fossil fuels [1]. Therefore, it has attracted political and business interest and extensive research is carried out at different universities and institutes [2].

It is very important to reduce the costs of design, manufacturing, and maintenance and optimize the power performance of wind turbines in order to be cost competitive in the current market. A better understanding and consequently improvement of wind turbine drive train dynamics performance could lead to overall cost efficiency in wind turbine operations. This is done by advanced system modelling, identifying the reasons for premature failures and fault diagnoses [3] in different components in drive train of wind turbines. Examples of multibody dynamic analysis to predict the components' loads in wind turbine drive trains are in
[4-6]. Generally, the dynamic performance of wind turbine drive train can be investigated based on different points of view, such as power performance, operational speeds, loads in specific components like coupling and bearings in both healthy and faulty situations, and misalignment parameters [7-14]. Different structural dynamic parameters can directly influence a wind turbine ultimate performance and in terms of loading, high speed shaft components are the most important since the components experience major extreme loads during different regimes, especially in transient one.

The current research deals with mathematical modelling of high speed shaft drive train test rig. A typical wind turbine drive train is composed of a generator as an electrical part with a control set, flexible couplings, a speed-up gearbox, a main shaft, and a wind rotor as an air-operated system [15]. The power from the rotor is transferred through main shaft, gearbox, and high speed shaft to the generator.

We propose the global sensitivity analysis (GSA) as a tool to better investigate wind turbine drive train dynamics, more 
specifically, high speed shaft drive train models. The aim is to quantify the input structural parameters' effect to objective functions of the system dynamics (interaction quantification set [16]). From GSA the most influential design parameters on objective functions of wind turbines can be determined, which could ease the optimization process by narrowing down the number of input design parameters.

Methodology of sensitivity analysis is various and it has been used for different applications. Reviews on different GSA methods can be found in [16-20]. Some examples of sensitivity analysis are derivative-based Morris one-at-atime (OAT) local sensitivity method [21] and Sobol/Saltelli method providing the variance based sensitivity [22, 23], whereas the Sobol/Saltelli variance based sensitivity method provides indices that quantify the relative contribution of each input structural parameter to set of objective functions $[22,23]$. In the variance based GSA, input variables are chosen randomly in a proper range. Then, the contribution of input parameter to objective functions is investigated via the sensitivity indices. These sensitivity indices are quantifying the contribution of a random variable to the total variance of the response, which is basis of variance based global sensitivity analysis in [23].

Computational effort when design analysis requires a large set of loading conditions to be evaluated, such as wind turbine drive trains, is very important. As one of the most common methods for GSA, Monte Carlo simulation for these systems is not efficient since it is computationally expensive. On the other hand, GSA within analysis of variance decomposition (ANOVA) or high dimensional model representation (HDMR) theory is suitable method in terms of being cost effective. It decomposes the response function of a high dimensional system into combination of low dimensional system set. This reduces the computational effort. A multiplicative dimensional reduction method (MDRM) for GSA was proposed by Zhang and Pandey [24] entailing simple representation of GSA indices. The method has proved to be as accurate as Monte Carlo with much less computational effort [22].

Several works have been done within sensitivity analysis of rigid and flexible multibody systems with respect to structural parameters such as mass, moment of inertia, stiffness, and damping values [25-28]. In [29] extended Fourier amplitude sensitivity test method was used and the results of a single turbine and a set of large group of turbines was compared by GSA. Sensitivity analysis to perform a large set of data analysis for a wind farm was reported in [30].

Considering that the faults occur in high speed shaft drive trains, this could be a subject of study [31,32]. The test rig for high speed shaft drive train as subsystem of wind turbine has been constructed in order to do experimental study and acquire data for validation of developed mathematical model. Another purpose of the test rig is to investigate strategies to detect faults. Early fault detection is important since it can reduce the cost of maintenance drastically.

Ultimately, by introducing faults and defects and different components of high speed shaft drive train, we investigate the detectability of faults and failures within GSA. Such an analysis could lead to fault diagnose in components of wind turbine drive train and prevent failures in early stages.

In the following sections, the proposed mathematical model of high speed shaft drive train test rig with the experimental setup at the lab is presented. Then, input variables and different operational scenarios and objective functions are introduced. Different sources of faults and failures such as defect in the bearing, backlash in the coupling, and torque ripple in the motor are introduced and modelled. Ultimately, the GSA indices for the validated model have been investigated. Results of GSA allow one to understand the priorities in contribution of system parameters for any specific objective function and lead one to focus on the areas of operation that show greatest opportunities for optimization. The outcome of the current research leads to useful information regarding wind turbine drive train dynamics behaviour on different operational scenarios which not only provide possibility to reduce the number of input parameters for optimization and speed up the process, but also give insight to design functional components in proper ranges and in a smarter way. In particular, the main aim of the paper is to apply the GSA for the proposed mathematical model of high speed shaft drive train including aforementioned fault sources. Quantifying the faults effects on the drive train objective functions could be applied as a methodology to contribute to virtual condition monitoring in order to detect and predict faults and prevent failures in early stages in different components of drive trains.

\section{High Speed Shaft Drive Train System Test Rig}

The most common wind turbine is of lateral (horizontal) axis type, which entails main supporting tower upon which sits a nacelle and rotor. Inside the nacelle sits a main shaft supported by bearings on which the rotor hangs. The main shaft is connected to a gearbox, which, in turn, is connected to the generator with control system (Figure 1). The high speed shaft subsystem is one part where faults in bearing are often observed.

To support experimental and numerical studies of wind turbine drive train dynamics, a scaled down test rig has been built and instrumented as reported in $[33,34]$. It entails a motor, rotor disk with shaft, where the rotor shaft is passing through the bearing housing and connected to the motor by a coupling. A small eccentric mass can be added on the rotor disk to give a varying load. The setup of the drive train test rig is represented in Figure 2.

The test rig is driven by ABB motor model M3BP160 MLA 6 (6 pole, $75 \mathrm{~kW}$ ) with a variable frequency controller (ACS355 from ABB providing speed up to $1000 \mathrm{rpm}$ ). In addition, the test rig entails motor shaft adapter, shaft's coupling, bearing housing with flexible support, a disk on which an eccentric mass can be added, and bedplate on which all the components are mounted.

The data acquisition system is SKF @ptitude Observer; SKF offers under its Windcon 3.0 condition monitoring system. The set of sensors comprise accelerometers and Eddy 


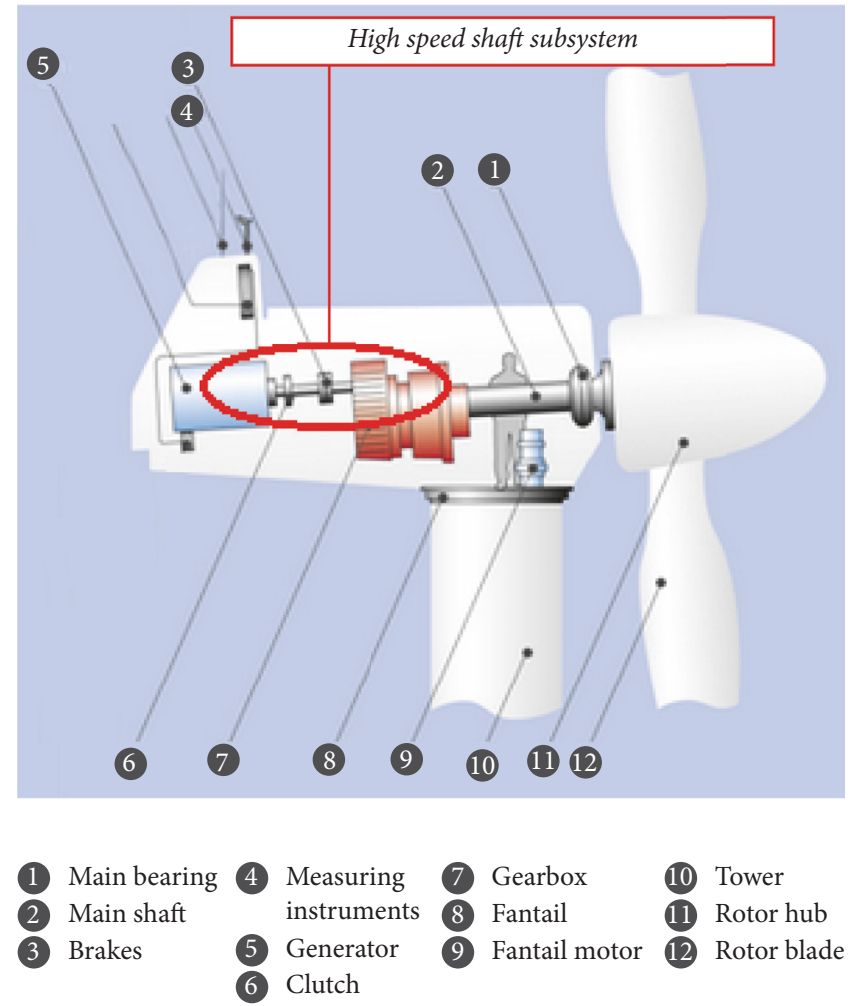

FIGURE 1: Overview of a high speed shaft subsystem as part of wind turbine drive train [52].

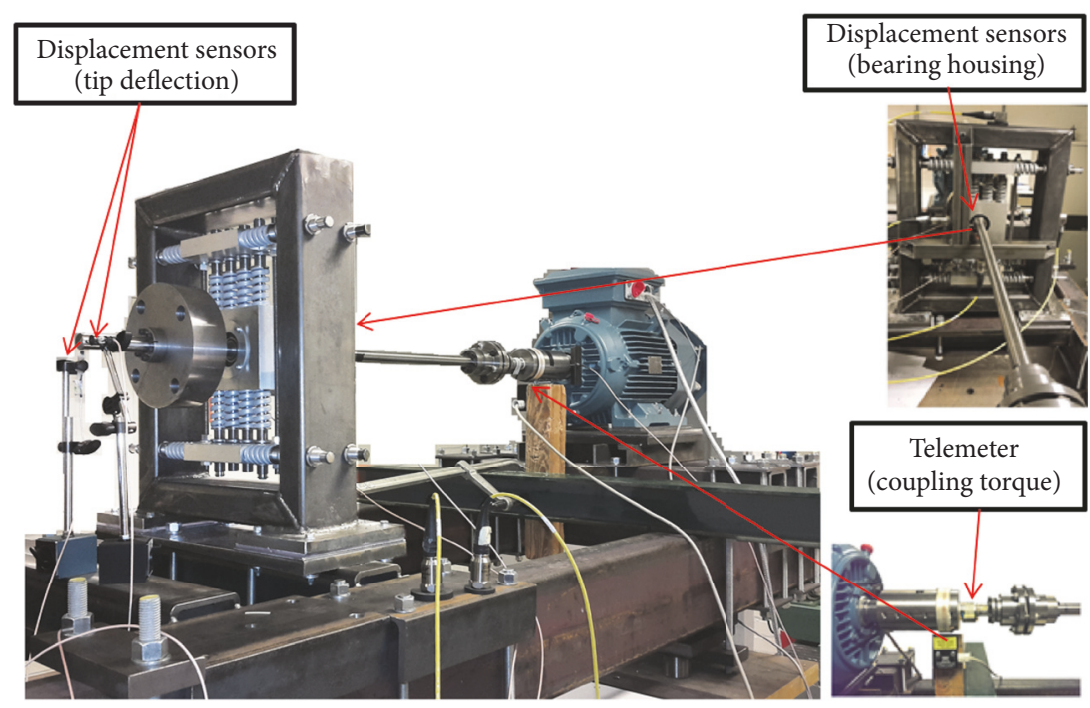

FIgURE 2: The test rig with sensors location.

probes displacement sensors, which detect vibrations in the test rig and deflection at both tip and bearing housing. Additionally, in order to measure the torque in coupling, full bridge strain gauges are put on the motor shaft, and the signal is acquired using a telemeter system (TEL 1-PCM).

In previous works $[33,34]$, the coupling torque, deflection fields in both lateral and vertical directions in tip, and bearing housing have been measured and used for model validation. To support future studies of defect detection, the objective functions derived in this paper are based on these sensors.

2.1. Mathematical Model of Drive Train System Test Rig. An engineering abstract of the test rig is shown in Figure 3. The mathematical model has been developed based 


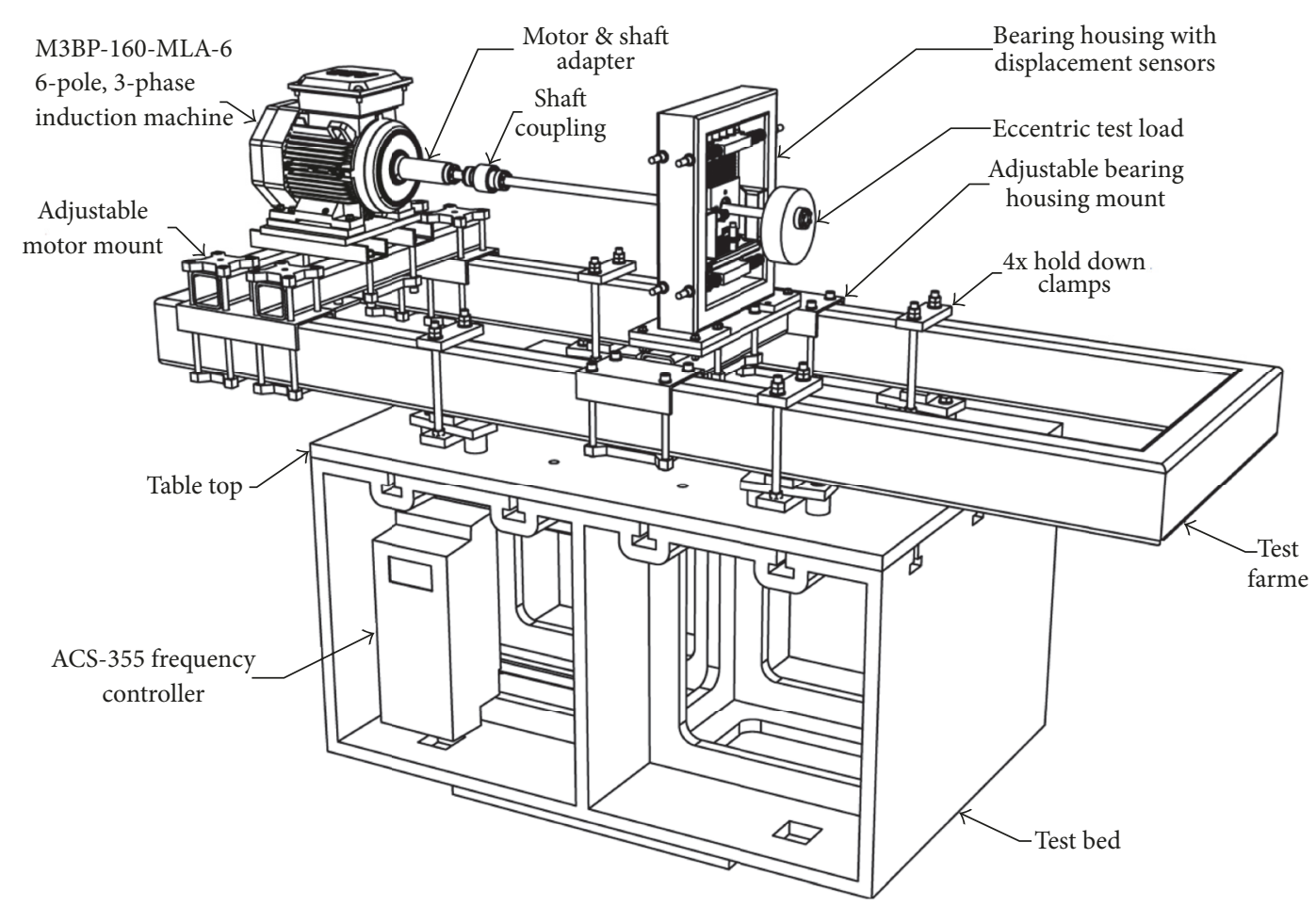

(a)

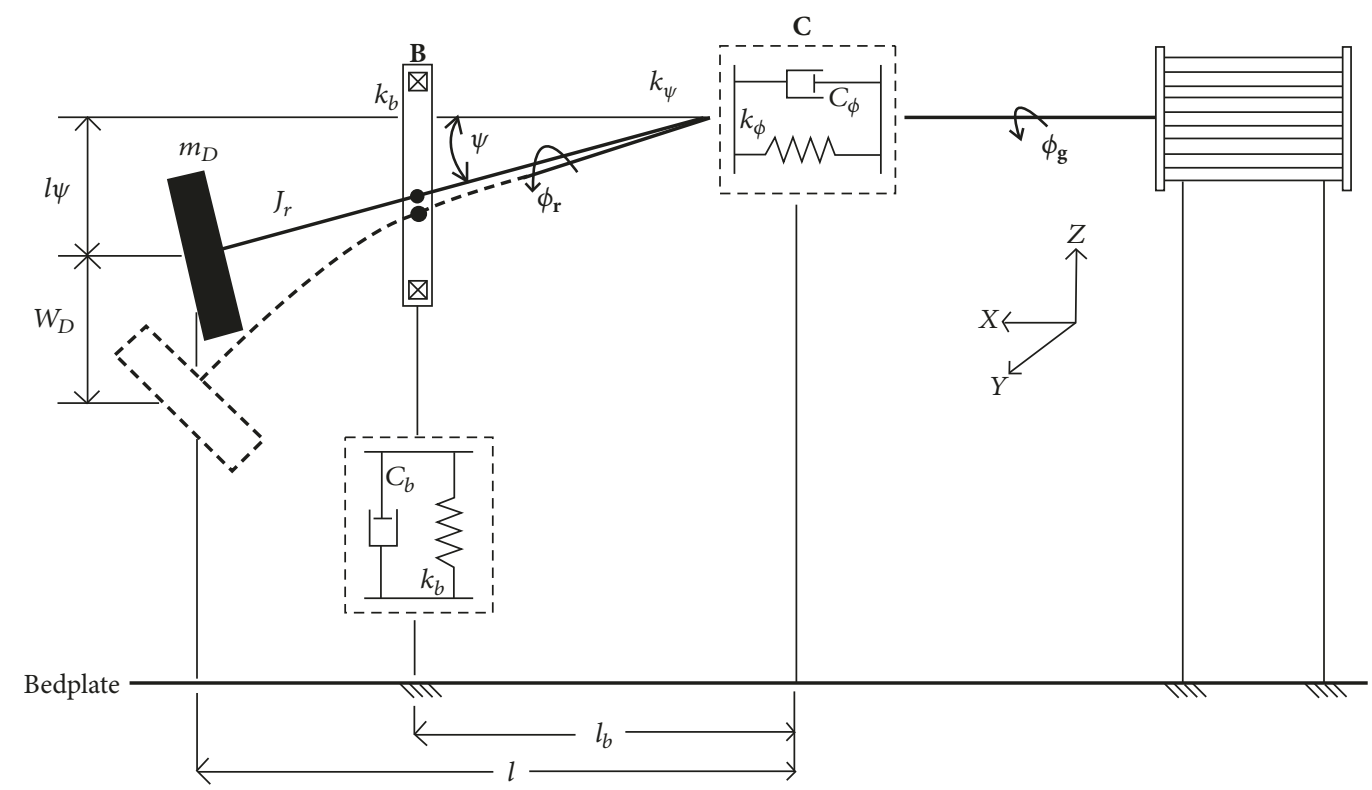

Rigid shaft

- - - Flexible shaft

(b)

FIGURE 3: A sketch of the test rig (a) and an engineering abstract (b).

on both rigid and flexible shaft assumption in bending modes, considering that the torsional flexibility is concentrated at coupling. The motor is rigidly attached to the bedplate.
2.1.1. Governing Equations. The kinetic, potential, and dissipative energies and nonconservative forces are derived considering that the shaft is rotating, and its bending deflection $w(x)$ is assumed to occur in the radial direction only. The 
disk is seen as a rigid body, and the shaft torsional flexibility is concentrated in coupling as well as linear along the shaft length. A linear spring model is adopted to simplify the flexibility of structure of the bearings [35].

$$
\begin{aligned}
T_{D} \doteq & \frac{1}{2} m_{D}\left[\left[\dot{w}_{D} \varphi^{\prime}(l)\right]^{2}+\left[w_{D} \varphi(l)\right]^{2} \dot{\phi}_{s}^{2}\right] \\
& +\frac{1}{2} m_{e}\left[\dot{w}^{\prime}(l)+\left(w(l)+r_{e}\right)^{2} \dot{\phi}_{s}^{2}\right]+\frac{1}{2} J_{D} \dot{\phi}_{D}^{2} \\
& +\frac{1}{2} I_{D}\left[\dot{w}_{D} \varphi^{\prime}(l)\right]^{2}, \\
T= & T_{D}+\frac{1}{2} J_{g} \dot{\phi}_{g}^{2}+\frac{1}{2}\left[J_{c}+\frac{J_{\text {sh }}}{2}\right] \dot{\phi}_{s}^{2}+\frac{1}{2}\left[\frac{J_{\mathrm{sh}}}{2}\right] \dot{\phi}_{D}^{2}, \\
V= & \left(m_{D}+m_{e}\right) g w(l) \cos \phi_{s}+\frac{1}{2} k_{\phi}^{\mathrm{sh}}\left(\phi_{D}-\phi_{s}\right)^{2} \\
& +\frac{1}{2} k_{\phi}^{C}\left(\phi_{s}-\phi_{g}\right)^{2}+\frac{1}{2} k_{b}^{\psi}\left[w_{D} \varphi\left(l_{b}\right)\right]^{2}+V_{\mathrm{sh}}, \\
R= & \frac{1}{2} C_{\phi}^{\mathrm{sh}}\left(\dot{\phi}_{D}-\dot{\phi}_{s}\right)^{2}+\frac{1}{2} C_{\phi}^{C}\left(\dot{\phi}_{s}-\dot{\phi}_{g}\right)^{2}+\frac{1}{2} C_{b f}\left|\dot{\phi}_{s}\right| \\
& +\frac{1}{2} C_{b}\left[\dot{w}_{D} \varphi\left(l_{b}\right)\right]^{2} .
\end{aligned}
$$

Here $V_{\text {sh }}=\int_{0}^{l} E I\left(w^{\prime \prime}(x)\right)^{2} d x$ is the contribution of the shaft bending to potential energy and the indices sh and $D$ denotes for shaft and disk, respectively. The complete list of structural parameters is given in Table 1. The shaft torsion is parametrized by $\phi_{D}, \phi_{s}$, and $\phi_{g}$, as an angle of rotation in the disk (rotor tip), shaft, and motor, respectively.

Applying Lagrange formalism, the nonlinear set of equations of motion can be written as matrix equation with time variant coefficients as follows:

$$
M(q) \ddot{q}+B(q, \dot{q}, t)=F(q, \dot{q}, t),
$$

where $M$ is the inertia matrix, $B$ is the vector related to internal forces acting on the test rig, and $q$ is the vector of generalized coordinate to be defined in Section 2.1.2.

$F$ is an external load vector and it is a combination of $M_{\mathrm{BH}}^{\bullet}(q(t)), T_{\mathrm{C}}^{\mathrm{BL}}(q(t))$, and $M_{g}^{\mathrm{rip}}(q(t))$ which applies to specific degree of freedom.

$M_{\mathrm{BH}}^{\bullet}$ corresponds to external forces due to the disturbance of the bearing defects imposed to the system (described in Section 2.2.3). The defect may impose the disturbance torque into bending and torsional degrees of freedom $(\bullet=$ $\left.\left\{\psi, \phi_{r}\right\}\right)$.

$T_{C}^{\mathrm{BL}}$ refers to the backlash of the coupling which appears as an internal load in the governing equations. The backlash has a contribution in the potential energy since it is modelled as a spring with gap.

$M_{g}^{\text {rip }}$ is torque ripples imposed by the motor, a source of faults in drive train dynamics representing electromechanical interaction between components.

2.1.2. Parametrization of the Model. Since the coupling is assumed to be very stiff in torsion (apart from its backlash), the shaft torsional flexibility and coupling play are here added and included in $k_{\phi}$, and $T_{C}^{\mathrm{BL}}$. We introduce $\phi_{r}$ as the rotor (disk plus shaft) angle, such that the shaft twist is represented by $\Delta \stackrel{\text { def }}{=} \phi_{g}-\phi_{r}$ where $\phi_{g}$ is the motor shaft angle. Hence, $\phi_{s}$ and $\phi_{D}$ are both replaced by $\phi_{r}$ in the governing equations in Section 2.1.1. We assume that the shaft inertia and coupling are abstracted as $J_{r}=J_{\text {sh }}+J_{C}$. Coupling torsional stiffness is represented in a way that includes both shaft and coupling torsional stiffness's. Similarly, the shaft and coupling damping, $C_{\phi}^{\text {sh }}$ and $C_{\phi}^{C}$, are represented by $C_{\phi}$.

The shaft deflection is parametrized in terms of rigid and flexible contribution to total deflection as $w(x)=\psi x+$ $w_{D} \varphi(x)$, where $\psi$ is the angular deflection at the coupling, $w_{D}$ is the rotor shaft tip deflection due to shaft flexibility, and $\varphi(x)$ is taken as the shape of the fundamental bending eigenmode as described in [33]. In [33], we developed and validated the mathematical model of high speed shaft drive train test rig considering the shaft flexibility in terms of bending. To obtain a suitable shape function $\varphi(x)$, the EulerBernoulli beam theory is applied [36]. Only radial vibration amplitudes of the beam were considered. The fundamental bending eigenmode based on structural parameters in Table 1 was found at $\beta=0.8734$ and its shape function $\varphi$ for a shaft rotating at speed $\Omega=1000 \mathrm{rpm}$ gives the eigenfrequency at $\omega^{\star}=109.4608 \mathrm{rad} / \mathrm{s}$. The next eigenmode was found at $\omega^{\star}=6151 \mathrm{rad} / \mathrm{s}$, which is much higher than relevant excitation frequency. The deflection fields in two locations, namely, tip $(x=l)$ and bearing hub $\left(x=l_{b}\right)$, are assessed within the aforementioned assumptions. Thus, the tip radial deflection would be $\psi l+w_{D} \varphi(l)$, and the bearing housing radial deflection becomes $\psi l_{b}+w_{D} \varphi\left(l_{b}\right)$. The displacements in vertical and lateral directions become mirrored by rotational angle $\phi_{r}$. Note that we simplify the deflection field in the bearing hub. For more realistic model, shaft prebending and relative motion of inner ring with respect to the bearing hub frame must be considered.

To conclude, the vector of generalized coordinate is defined as $q=\left[\begin{array}{llll}\psi & \phi_{r} & \phi_{g} & w_{D}\end{array}\right]$.

\subsection{Faults Modelling in the Drive Train}

2.2.1. Electromechanical Modelling of the Motor: Motor Torque with Ripple. Considering that wind turbine drive train contains both mechanical and electrical components, it is of important concern to have a model which studies electromechanical interactions. In induction motors, many kinds of excitation occur such as electromagnetic forces exist, that is, unbalanced magnetic pull. Torsional vibration of drive train system could lead to significant fluctuation of motor speed. Such oscillation of the angular speed superimposed on the average motor angular speed causes severe oscillation of electric currents in motor windings [37]. In this section, we model torque ripple as source of this fluctuations in motor speed.

In the current mathematical model, we assume the motor as a 6-pole induction machine with rated power $7.5 \mathrm{~kW}$ at speed $975 \mathrm{rpm}$. The speed is set from a frequency converter, such that the torque is given by the difference from set speed 
TABLE 1: Test rig nominal and randomized input parameters.

\begin{tabular}{|c|c|c|c|c|}
\hline Parameter & Symbol & $\begin{array}{c}\text { Nominal value } \\
\left(X^{\star}\right)\end{array}$ & $X_{\min } / X^{\star}$ & $X_{\max } / X^{\star}$ \\
\hline Motor inertia with respect to $x$ axis & $J_{g}$ & $0.087 \mathrm{kgm}^{2}$ & 0.5 & 2 \\
\hline Rotor inertia with respect to $x$ axis & $J_{r}$ & $0.095 \mathrm{kgm}^{2}$ & 0.5 & 2 \\
\hline Rotor shaft inertia with respect to $x$ axis & $J_{\text {sh }}$ & $0.0003 \mathrm{kgm}^{2}$ & & \\
\hline Rotor inertia with respect to $\psi$ (minor axis) & $I_{r}$ & $19.083 \mathrm{kgm}^{2}$ & 0.5 & 2 \\
\hline Disk inertia with respect to $y$ axis & $I_{D}$ & $18 \mathrm{kgm}^{2}$ & & \\
\hline Shaft inertia with respect to $y$ axis & $I_{\mathrm{sh}}^{Y}$ & $0.5 \mathrm{kgm}^{2}$ & & \\
\hline Disk mass & $m_{D}$ & $14.52 \mathrm{~kg}$ & 0.75 & 1.5 \\
\hline Rotor shaft mass & $m_{\mathrm{sh}}$ & $3.85 \mathrm{~kg}$ & & \\
\hline Eccentric mass value mounted in the rotor disk & $m_{e}$ & $74.4 \mathrm{gr}$ & 0 & 4 \\
\hline Distance of eccentric mass from center & $r_{e}$ & $8 \mathrm{~cm}$ & & \\
\hline Rotor shaft and disk mass & $m_{r}$ & $18.35 \mathrm{~kg}$ & & \\
\hline Length from coupling to the tip & $l$ & $0.85 \mathrm{~m}$ & 0.8 & 1.2 \\
\hline Length from coupling to bearing housing & $l_{b}$ & $0.72 \mathrm{~m}$ & 0.8 & 1.2 \\
\hline Length from coupling to CG of disk and rotor shaft & $l_{0}$ & $0.75 \mathrm{~m}$ & & \\
\hline Damping factor & $\zeta_{d}$ & $0.01 \mathrm{~s}$ & & \\
\hline Coupling torsional stiffness & $k_{\phi}$ & $2600 \mathrm{Nm} / \mathrm{rad}$ & 0.75 & 5 \\
\hline Coupling bending stiffness & $k_{\psi}$ & $56 \mathrm{kNm} / \mathrm{rad}$ & 0.75 & 5 \\
\hline Bearing mount lateral and vertical stiffness coefficients & $k_{b}^{0}$ & $4308 \mathrm{kN} / \mathrm{m}$ & 0.75 & 5 \\
\hline Bearing hub bending stiffness & $k_{b}^{\psi}$ & $0.1 k_{\psi}$ & 0.75 & 5 \\
\hline Coupling torsional damping & $C_{\phi}$ & $26 \mathrm{kNm} / \mathrm{rad}$ & 0.5 & 10 \\
\hline Coupling bending damping & $C_{\psi}$ & $56 \mathrm{kNm} \mathrm{s} / \mathrm{rad}$ & 0.5 & 10 \\
\hline Bearing mount lateral and vertical damping coefficients & $C_{b}$ & $4308 \mathrm{kN} \mathrm{s} / \mathrm{m}$ & 0.5 & 10 \\
\hline Bearing friction damping coefficient & $C_{b f}$ & $0.0023 \mathrm{Ns} / \mathrm{m}$ & 0.8 & 3 \\
\hline Rotor torsional damping coefficient plus bearing friction & $C_{b r}^{f}$ & $0.006 \mathrm{Ns} / \mathrm{m}$ & 0.8 & 3 \\
\hline Motor torsional damping coefficient & $C_{b g}$ & $0.006 \mathrm{Ns} / \mathrm{m}$ & 0.5 & 10 \\
\hline Shaft bending stiffness & $E I$ & $7.6699 \mathrm{kNm}^{2}$ & 0.5 & 10 \\
\hline Angle dependency factor of $k_{b}$ & $\alpha_{b}$ & 1 & 0.8 & 1.5 \\
\hline Torque ripples parameters & $M_{\text {rip }}^{(i)}$ & $10 \mathrm{Nm}$ & 0 & 3 \\
\hline Backlash gap & $\mathrm{BL}$ & $0.002 \mathrm{~m}$ & 0 & 3 \\
\hline Backlash regularization parameter & $\sigma$ & 40 & & \\
\hline Bearing torque disturbance zone for defect & $\varepsilon$ & 0.1 & & \\
\hline Maximum value of rotational bearing defect torque & $M_{\mathrm{BH}}^{\phi_{r}}$ & $30 \mathrm{Nm}$ & 0 & 5 \\
\hline Maximum value of radial bearing defect torque & $M_{\mathrm{BH}}^{\psi}$ & $30 \mathrm{Nm}$ & 0 & 5 \\
\hline
\end{tabular}

$\omega_{g}^{\text {set }}$ and actual speed $\dot{\phi}_{g}$. A cycle of ramp startup-steady stateshutdown can thus be described by

$$
M_{\text {mech }}=\frac{M_{\text {mark }}}{\Delta \omega_{\text {mark }}}\left(\omega_{g}^{\text {set }}-\dot{\phi}_{g}\right) \text {, }
$$

where

$$
\begin{aligned}
\omega_{g}^{\text {set }} & =\frac{t}{t_{0}} \omega_{g}^{\max } \quad t<t_{0} \\
\omega_{g}^{\text {set }} & =\omega_{g}^{\max } \quad t_{0} \leq t<t_{1} \\
M_{\text {mark }} & =0 \quad t_{1} \leq t,
\end{aligned}
$$

where $\Delta \omega_{\text {mark }}=25 / 60 \times 2 \pi \mathrm{rad} / \mathrm{s}, M_{\text {mark }}=73.4561 \mathrm{Nm}, t_{0}$ is the startup-time, and $t_{1}$ is the instance of time when motor is turned off (without braking). From electrical measurement of the voltage from the frequency converter, it was found that, in addition to $M_{\text {mech }}$, there is a small periodic torque (ripple) acting on the generator rotor at frequencies $\omega_{g}^{\text {set }}, 3 \omega_{g}^{\text {set }}$, and $6 \omega_{g}^{\text {set }}$. Hence, the following electrical torque is considered:

$$
\begin{aligned}
M_{g}(t)= & \frac{M_{\text {mark }}}{\Delta \omega_{\text {mark }}}\left(\omega_{g}^{\text {set }}-\dot{\phi}_{g}\right)+M_{\text {rip }}^{(1)} \sin \left(\phi_{g}\right) \\
& +M_{\text {rip }}^{(2)} \sin \left(3 \phi_{g}\right)+M_{\text {rip }}^{(3)} \sin \left(6 \phi_{g}\right),
\end{aligned}
$$

where $M_{\text {rip }}^{(i)}(i=\{1,2,3\})$ are coefficients that contribute to torque ripple with corresponding frequencies and have been obtained based on comparison of the torque ripple range in steady state in simulation and experiments. 


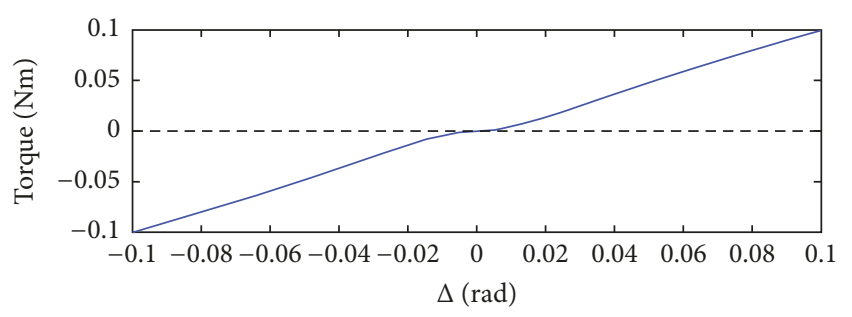

FIgURE 4: Backlash effect as a torque to the coupling.

2.2.2. Backlash Modelling of the Coupling. Here, we propose backlash as nonlinear stiffness properties to the system structure, which is common source of faults in transmission system dynamics (Figure 4). This phenomenon affects the governing equations. Properly functioning mechanical systems need to have a certain clearance (gap, play) between the components transmitting motion under load. As part of internal forces, $T_{\mathrm{int}}$ is defined as follows [38]:

$$
\begin{aligned}
T_{\text {int }}(\Delta)= & k_{\phi}(\Delta-\mathrm{BL})+\left(k_{\phi}-\mathrm{BL}\right) \\
& \times \frac{[g(\Delta-\mathrm{BL})-g(\Delta+\mathrm{BL})]}{2}, \\
g(x)= & |x| \tanh (\sigma x) .
\end{aligned}
$$

2.2.3. Modelling of Defects in Rolling Elements (Bearing): Disturbance Torque from Bearing Inner Ring. Bearing fault diagnosis is important in condition monitoring of any rotating machine. Early fault detection is one of the concerns within the wind turbine system modelling. To this end, we need to model source of faults in the bearing. Dynamic modelling and vibration response simulation of rolling bearings with surface defects is a common assumption within fault modelling. When bearing balls roll over defects, shock pulses with high frequency are generated with excessive vibration leading the whole system to failure. This characteristic impulse-response signal is detected by the vibration monitor. An inner raceway rotates with shaft's rotation and goes through the loaded zone of bearing every rotation. This mechanism shows that the vibration of inner raceway with defect is modulated by the shaft rotation [39-41].

Here we propose a simple modelling of surface defect for bearing which imposes a disturbance torque in certain angles in the inner raceway connected to the rotor shaft (similar to when a ball passes from the defects and causes a excitation to the system). Figure 5 shows the external torque imposed in radial and rotational directions. As part of external forces, $M_{\mathrm{BH}}^{\bullet}$ is defined based on impulse factor $\varepsilon$ and maximum torque $M_{\mathrm{BH}}^{\cdot}$ as shown in Figure 5(a).

Proper value for maximum disturbance is $M_{\mathrm{BH}}^{\max }=$ $30 \mathrm{Nm} . \varepsilon(=0.1)$ is the angular length of active contact zone, where the defect is assumed (Figure 5).

Depending on the rolling elements number included in the bearing structure, the geometric relationship among its components, and shaft rotational speed, characteristic rotational frequencies initiating from respective bearing elements could be obtained. In this regard, the characteristic passing frequency in the inner ring is defined as follows:

$$
f_{i}=f_{r} \frac{N_{b}}{2}\left(1+\frac{D_{b}}{D_{c}} \cos \varphi\right),
$$

where $f_{r}$ is the rotor rotational frequency $\left(=\dot{\phi}_{r} / 2 \pi\right), N_{b}$ is number of rolling balls $\left(N_{b}=9\right), D_{b}(=72 \mathrm{~mm})$ and $D_{c}(=53.5 \mathrm{~mm})$ are the balls diameter and bearing pitch $\left(\left(D_{o}+\right.\right.$ $\left.\left.D_{i}\right) / 2\right)$ diameters, respectively. $\varphi$ is the contact angle $(\varphi=$ $0 \mathrm{rad}$ ). The bearing defect location (here, the inner race) will determine that $f_{i}\left(\approx 10.55 f_{r}\right)$ will be exhibited in the machine vibration signal.

2.2.4. Uneven Mounting Stiffness. For subsequent study of excitation of uneven mounting stiffness, the bearing mount stiffness has been defined as function of rotor shaft angle $\phi_{r}(t)$ as follows:

$$
k_{b} \doteq k_{b}^{0} \sqrt{\cos ^{2} \phi_{r}+\frac{1}{\alpha_{b}} \sin ^{2} \phi_{r}}
$$

where $\alpha_{b}$ is a parameter corresponding to angle dependency of $k_{b}$ and defines different loads in vertical and lateral directions imposed to the bearing springs.

2.3. Numerical Values of Parameters in the High Speed Shaft Drive Train Test Rig. Table 1 represents the parameters and their numerical values assumed in the mathematical model:

The damping coefficients are chosen as proportional to respective stiffness coefficient: $C_{\bullet} \doteq \zeta_{d} k_{\bullet}$, where $\bullet=\{\phi, \psi, b\}$.

The value of $C_{b f}$ which represents the bearing friction damping has been defined from SKF manual handbook [42].

2.4. Operational Scenarios. In order to study the GSA in different conditions, we introduce the following set of operational scenarios in wind turbine drive train dynamic analysis such as transient, steady state, and shutdown:

(i) Transient: a torque is applied to reach the preset speed.

(ii) Steady state: it is where the structure is under almost constant speed control.

(iii) Shutdown: in this regime there is no torque imposed to the system, and it is left to slow down to stop.

Note that the corresponding motor speeds ([1000 800600$] \mathrm{rpm}$ ) correspond to the specific frequency in the frequency controller ([ [ [ $\left.\begin{array}{lll}50 & 40 & 30\end{array}\right] \mathrm{Hz}$, resp.).

2.5. System Model Response for the Nominal Values. Here, we present the simulation response of the mathematical model based on structural parameters nominal values represented in Table 1. The displacement fields in both lateral and vertical direction with their FFT have been presented.

The deflection fields include three different operational scenarios based on Figure 6 with steady state of $t=\left[\begin{array}{ll}1 & 6\end{array}\right] \mathrm{sec}$ and shutdown starting at $t=6 \mathrm{sec}$. The tip deflection fields in both vertical and lateral directions are presented with zoom 


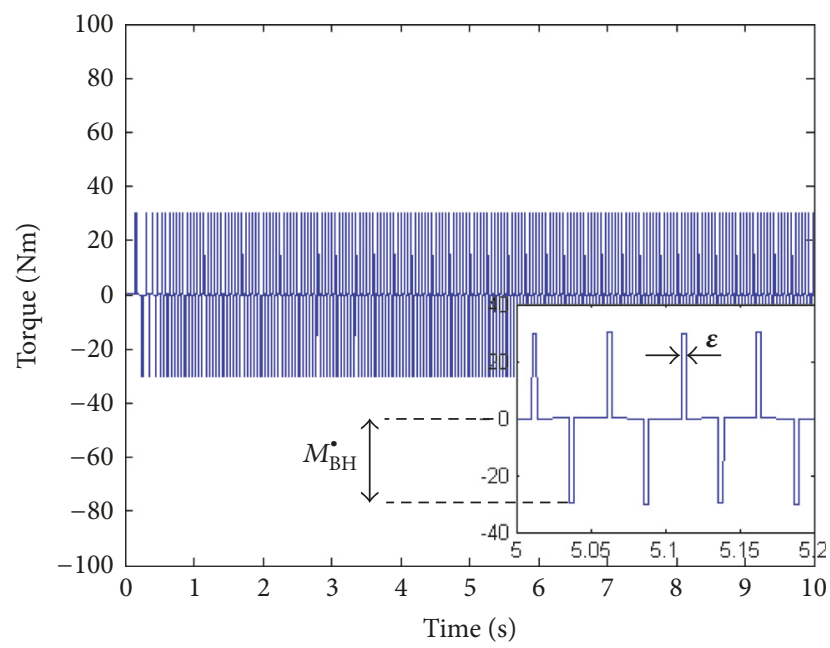

(a)

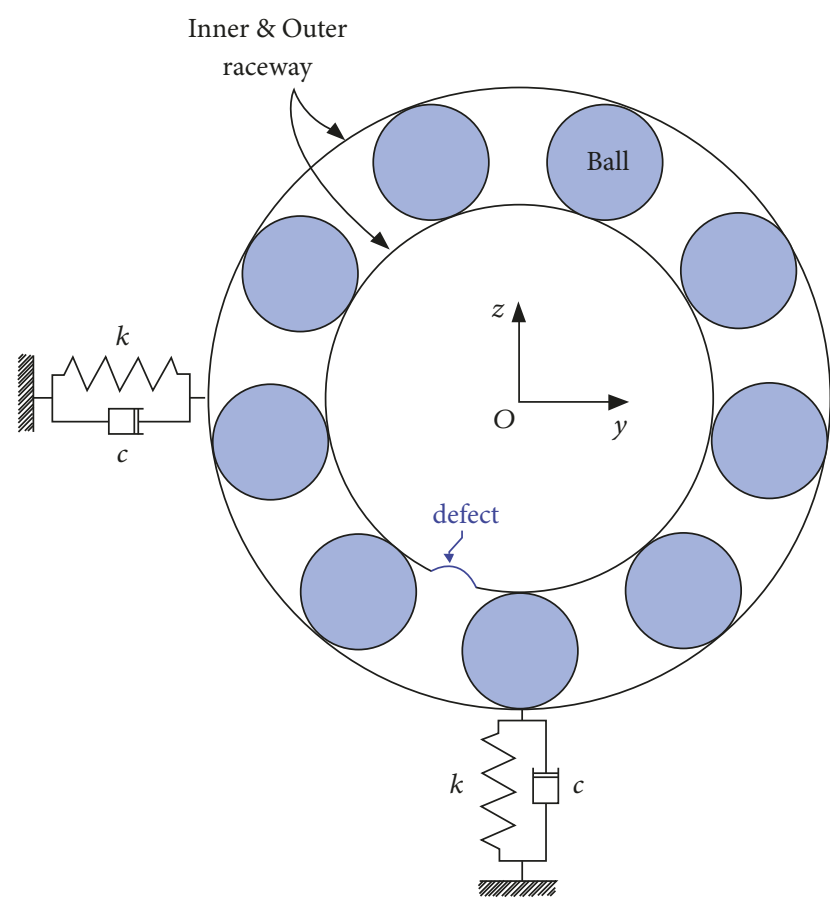

(b)

FIgURE 5: Typical disturbance torque $\left(M_{\mathrm{BH}}^{\cdot}\right)$ with zoom (a) and inner race defect modelling sketch with rolling bearing elements (b).

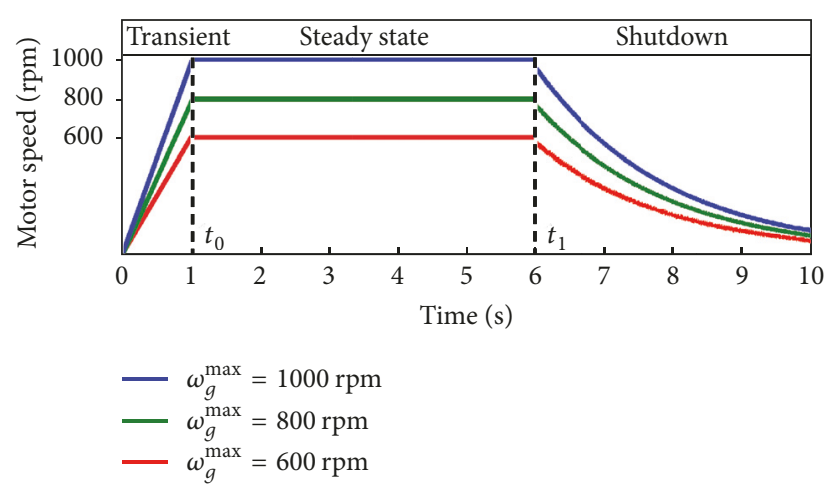

FIGURE 6: Motor speed for operational scenarios transient, steady state, and shutdown for 3 different maximum speeds.

in steady state $\left(t=\left[\begin{array}{ll}5 & 6\end{array}\right] \mathrm{sec}\right)$ and shutdown $\left(t=\left[\begin{array}{ll}7 & 8\end{array}\right] \mathrm{sec}\right)$ in Figure 7.

The bearing housing deflection fields in both vertical and lateral directions are presented with zoom in steady state $\left(t=\left[\begin{array}{ll}5 & 6\end{array}\right] \mathrm{sec}\right)$ and shutdown $\left(t=\left[\begin{array}{ll}7 & 8\end{array}\right] \mathrm{sec}\right)$ are shown in Figure 8 .

The frequency response functions of the tip deflection in both vertical and lateral directions are presented with zoom on the dominant frequency in Figure 9.

The coupling torque with its frequency response is presented here in Figure 10.

The equations of motion have been solved using Newmark numerical procedure method implemented in Matlab software. In order to satisfy the convergence criteria, the proper time step has been chosen as $\Delta t=10^{-4}$.

\section{Objective Functions}

In this section we introduce the set of objective functions. The introduced objective functions are assessed in order to evaluate the dynamic performance of the developed high speed shaft models.

(i) Tip Deflection in Both Vertical and Lateral Directions in Steady State and Shutdown Regimes $\left(\mathrm{OF}_{B}^{1-4}\right)$. The RMS values of the tip deflection in both lateral and vertical in two operational scenarios (steady state and shutdown) $\left(\operatorname{RMS}\left(Y^{\text {std }}\right), \operatorname{RMS}\left(Z^{\text {std }}\right)\right.$ and $\operatorname{RMS}\left(Y^{\mathrm{SD}}\right), \operatorname{RMS}\left(Z^{\mathrm{SD}}\right)$, resp. $)$ are obtained within the mathematical model and the sensitivity indices based on the structural inputs have been defined for these objective functions.

(ii) Bearing Hub Deflection in Both Vertical and Lateral Directions in Steady State and Shutdown Regimes $\left(\mathrm{OF}_{B}^{5-8}\right)$. RMS value of the bearing housing bending in both lateral and vertical directions in two operational scenarios (steady state and shutdown) $\left(\mathrm{RMS}\left(\mathrm{BH}_{Y}^{\text {std }}\right), \mathrm{RMS}\left(\mathrm{BH}_{Z}^{\text {std }}\right)\right.$ and $\mathrm{RMS}\left(\mathrm{BH}_{Y}^{\mathrm{SD}}\right)$, $\mathrm{RMS}\left(\mathrm{BH}_{Z}^{\mathrm{SD}}\right)$, resp.) is obtained within the mathematical model and the sensitivity indices based on the structural inputs have been defined for these objective functions. A model of gearbox is required to consist of shaft and bearing housing stiffness, which allows interaction between housing and the internal components to be investigated. 


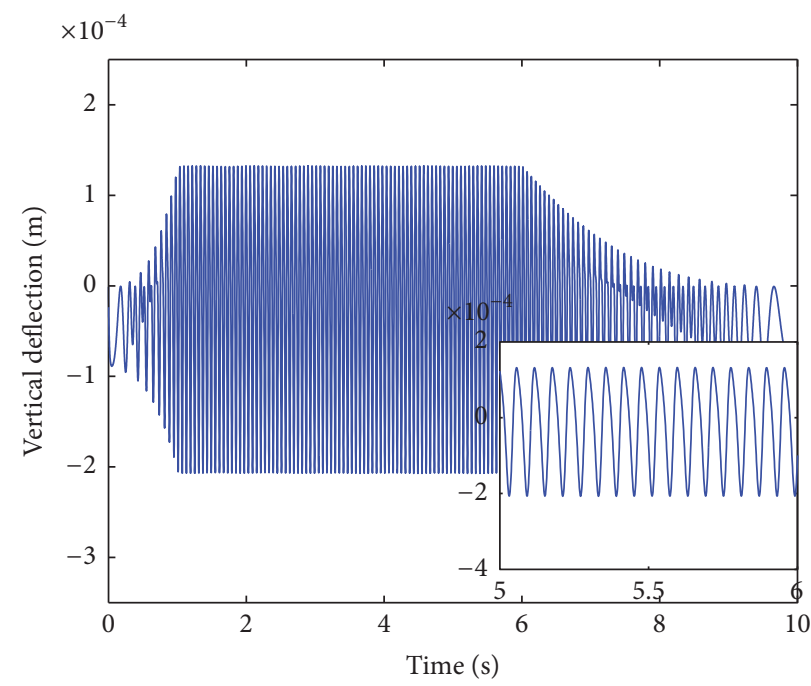

(a)

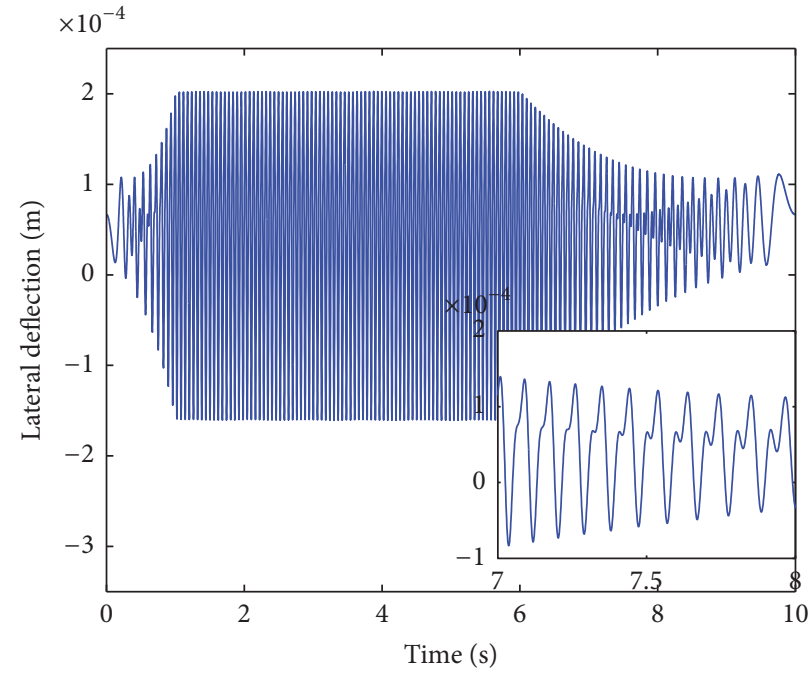

(b)

FIGURE 7: Tip deflection with zoom in steady state in vertical (a) and with zoom in shutdown regime in lateral (b) directions for the total model.

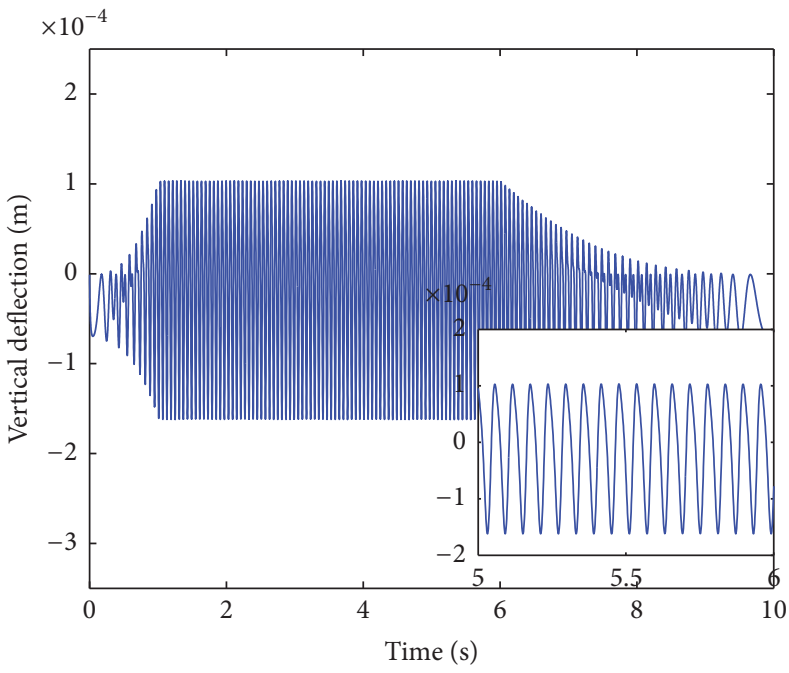

(a)

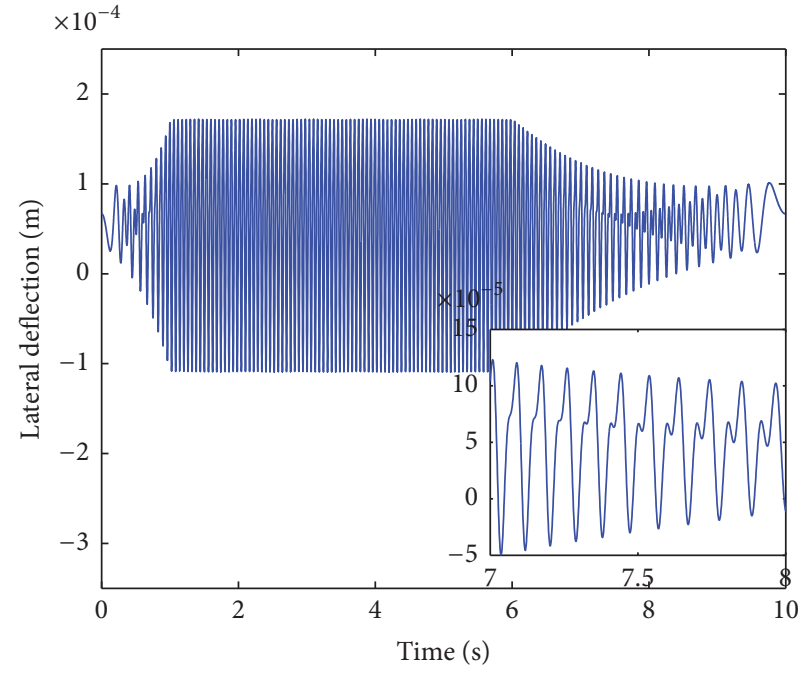

(b)

Figure 8: Bearing housing deflection with zoom in steady state in vertical (a) and with zoom in shutdown regime in lateral (b) directions for the total model.

(iii) Frequency Response of the Tip Deflection in Lateral and Vertical Directions in Steady State and Shutdown $\left(O F_{B}^{9-12}\right)$. These are the desired objective function representations: $\operatorname{FFT}\left(Y^{\text {std }}\right), \operatorname{FFT}\left(Z^{\text {std }}\right)$ and $\operatorname{FFT}\left(Y^{\mathrm{SD}}\right), \operatorname{FFT}\left(Z^{\mathrm{SD}}\right)$, respectively. The modal sensitivity analysis allows one to recognize the eigenfrequency response to design parameters changes. It can also help for the better identification of important dynamic properties.

(iv) Coupling Torque in Different Operational Scenarios Transient, Steady State, and Shutdown Cases $\left(\mathrm{OF}_{T}^{1-3}\right)$. In order to determine the influence of the design parameters in torsional vibration model, RMS values of the coupling torque in different operational scenarios transient, steady state, and shutdown cases are investigated as objective functions (RMS $\left(\mathrm{CT}^{\mathrm{tr}}\right)$, RMS $\left(\mathrm{CT}^{\mathrm{std}}\right)$, and RMS $\left(\mathrm{CT}^{\mathrm{SD}}\right)$, resp.). The coupling torque is defines as $M_{\phi}=k_{\phi} \Delta$.

Coupling torque in transient regime is very important to measure and quantify the parameters which have the most effect, since the component experiences the most severe loadings due to this regime nature. By shutting down the motor, there is not any load imposed by motor side and the range of the coupling torque is decreased from steady state value to zero in short period of time. 


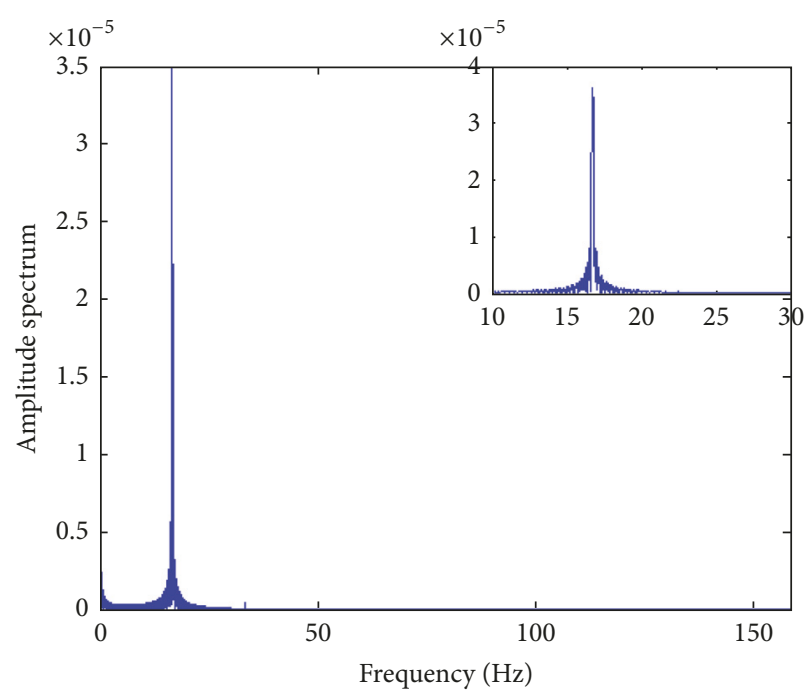

(a)

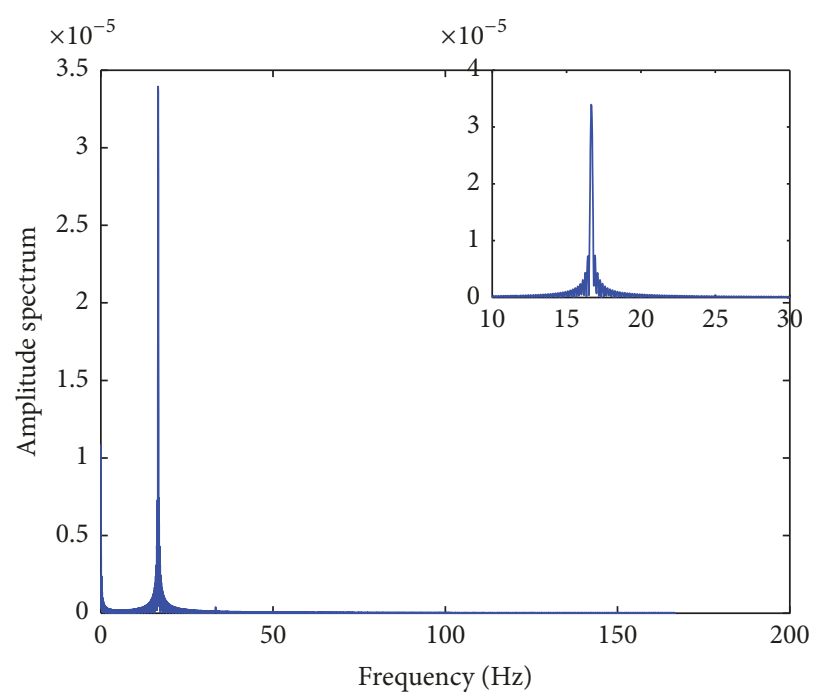

(b)

FIGURE 9: FFT of tip deflection in steady state regime, in vertical (a) and lateral (b) directions for the total model.

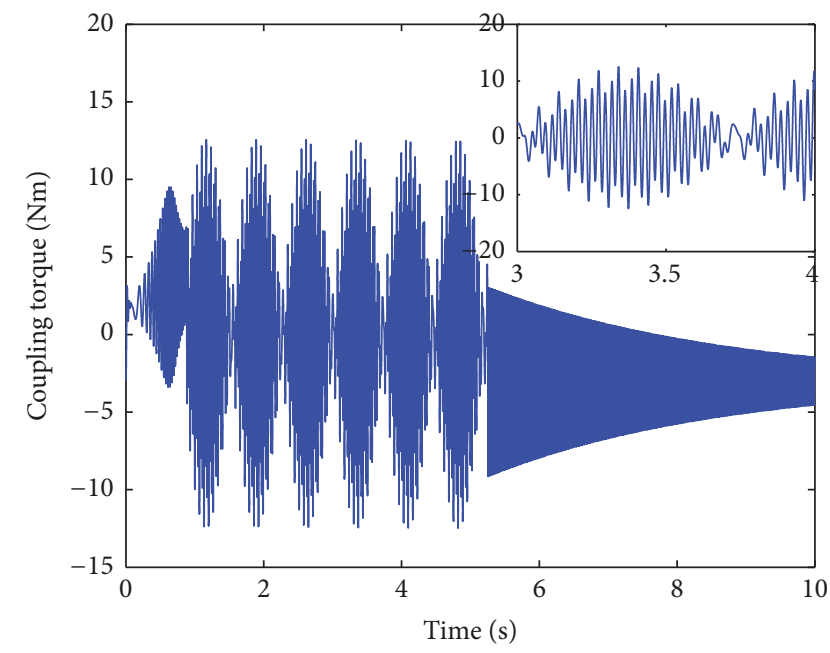

(a)

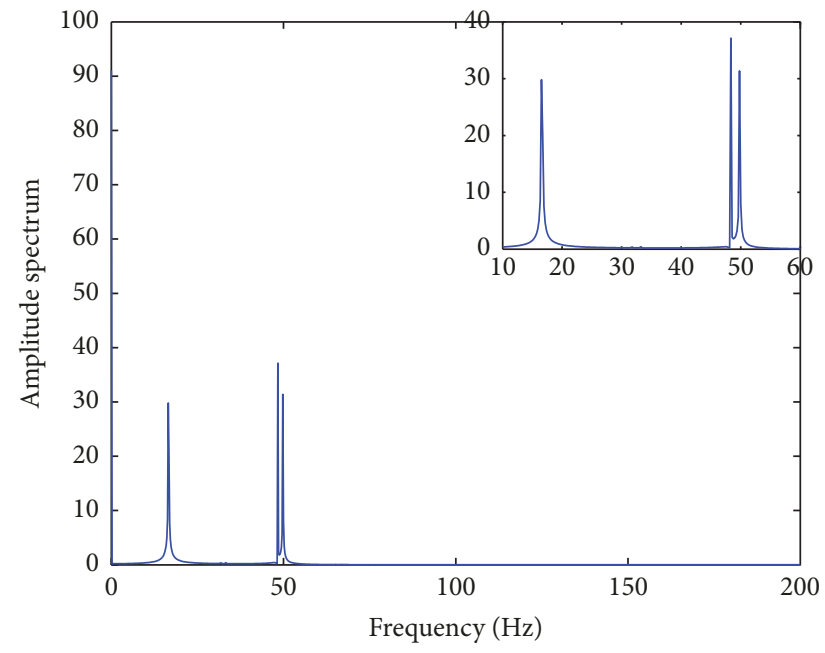

(b)

FIGURE 10: Coupling torque with zoom in the steady state (a) and its FFT with zoom in the peaks (b) for the total model.

(v) The First and Second Peaks in FFT Diagram of Coupling Torque in Steady State $\left(\mathrm{OF}_{T}^{4-5}\right)$. There are several picks in the coupling torque frequency response, of which the most dominant one is of interest to study, and investigating the most influential parameter would give valuable conclusion form the vibrational point of view. Studying the torsional model, we came up with a conclusion that some peaks in FFT are related to the ripple due to motor. Quantifying those peaks would give the contribution of the fault source to the objective function, which is very valuable information in terms of optimization.

(vi) The Peak in FFT Diagram of Coupling Torque in Shutdown Regime $\left(O F_{T}^{6}\right)$. During shutdown we expect that only torsional eignefrequency of the structure plays the role in FFT.

\section{Global Sensitivity Analysis of High Speed Shaft Test Rig}

The efficiency and applicability of global sensitivity analysis is already proven by some mathematical and mechanical examples [24]. The aim here is to apply this method to the high speed shaft drive train dynamics with respect to the structural parameters.

4.1. Theoretical Concept of Variance Based Sensitivity Analysis. Each of the objective functions in Section 3 can be seen as a function of the test rig structural parameters, represented as vector of $n$ variables, $\mathbf{X}=\left[X_{1}, X_{2}, \ldots, X_{n}\right]^{T}$. We have adopted the variance based sensitivity indices developed by Saltelli et al. [43] and mainly founded on the previous work of the Russian mathematician Zhang and Pandey [22]. These 
methods are defined as global sensitivity analysis (GSA), since the set of sensitivity indices are calculated considering the entire range of variations in parameters, compared to OAT (once at a time) or differential approaches [44].

Upon considering the structural parameters $\mathbf{X}$ as random, the objectives OF $=h(\mathbf{X})$ are also random. The expectation operation is denoted by $E[\bullet]$, such that the mean and variance of $h$ are calculated as follows:

$$
\begin{aligned}
& \mu_{\mathrm{OF}}=E[h(\mathbf{X})], \\
& V_{\mathrm{OF}}=E\left[\left(h(\mathbf{X})-\mu_{\mathrm{OF}}\right)^{2}\right] .
\end{aligned}
$$

Under certain assumptions of independence of $X_{i} \mathrm{~s}$, the ANOVA decomposition of $h(\mathbf{X})$ can be expressed as follows $[45,46]$ :

$$
\begin{aligned}
h(\mathbf{X})= & h_{0}+\sum_{i=1}^{n} h_{i}\left(X_{i}\right)+\sum_{i<j} h_{i j}\left(X_{i}, X_{j}\right)+\cdots \\
& +h_{12 \cdots n}\left(X_{1}, X_{2}, \ldots, X_{n}\right) .
\end{aligned}
$$

Upon taking the variance of (10), the total variance of OF can be decomposed in the same manner [47]:

$$
V_{\mathrm{OF}}=\sum_{i=1}^{n} V_{i}+\sum_{i<j} V_{i j}+\cdots+V_{12 \cdots n}
$$

where it was assumed that the components of the function (11) are orthogonal and can be expressed in terms of $h(\mathbf{X})$ integrals as

$$
\begin{aligned}
V_{i_{1} \cdots i_{s}} & =\int\left(h_{i_{1} \cdots i_{s}}\right)^{2} d x_{i_{1}} \cdots d x_{i_{s}}, \\
V_{\mathrm{OF}} & =\int(h)^{2} d \mathbf{X}-\left(h_{0}\right)^{2} .
\end{aligned}
$$

In case $X_{i}$ 's are not uniformly distributed, it is possible to define a probability function for random variables $X_{1}, X_{2}, \ldots, X_{s}$ and adjust (12) and (13) accordingly [22].

The constants $V_{\mathrm{OF}}$ and $V_{i_{1} \ldots i_{s}}$ are called variances of $h$ and $h_{i_{1} \ldots i_{s}}$, respectively. The first-order terms $V_{i}$ represent the partial variance in the response due to the individual effect of a random variable $X_{i}$, while the higher order terms show the interaction effects between two or more random variables. This decomposition puts forward two concepts, namely, the primary effect referring to the effect of a term associated with only one random variable [48] and the total effect referring to both the individual effect of a random variable as well as its interaction with other random variables. The primary effect index of a random variable $X_{i}$ is obtained by the normalization of the main-effect variance over the total variance in $\mathrm{OF}$ :

$$
S_{i}=\frac{V_{i}}{V_{\mathrm{OF}}}
$$

and the total sensitivity index is defined as

$$
S_{T_{i}}=\frac{V_{T_{i}}}{V_{\mathrm{OF}}},
$$

where $V_{T_{i}}=V_{\mathrm{OF}}-V_{-i}\left[E_{i}\left(\mathrm{OF} \mid \sim X_{i}\right)\right]$. Note that $V_{-i}[\bullet]$ refers to the variance of quantity with randomized set of $\mathbf{X}$, except $X_{i}$ taken as nominal value. More elaborate derivation of equations is presented in [22].

4.1.1. Multiplicative Dimensional Reduction Method (MDRM): To Compute the Simplified Sensitivity Indices. In order to reduce the computational effort associated with the numerical evaluation of (13), [22], we propose to utilize a multiplicative form of dimensional reduction method (MDRM) for sensitivity analysis, of which the corresponding deterministic model, $\mathrm{OF}=h(X)$, is written with reference to a fixed input point, that is, $\mathbf{X}=\mathbf{c}$, referred to as the cut point with coordinates $\mathbf{c}$. To this end, the $i$ th invariance functions defined by fixing all input variables, but $X_{i}$, to their respective cut point coordinates are derived as

$$
h_{i}\left(X_{i}\right)=h\left(c_{1}, \ldots, c_{i-1}, X_{i}, c_{i+1}, \ldots, c_{n}\right) .
$$

Based on this concept, a multiplicative dimensional reduction method approximates the deterministic function $h(\mathbf{X})$ as follows [24]:

$$
h(\mathbf{X}) \approx[h(\mathbf{c})]^{1-n} \cdot \prod_{i=1}^{n} h_{i}\left(X_{i}, \mathbf{c}_{-i}\right),
$$

where $h(\mathbf{c})=h\left(c_{1}, c_{2}, \ldots, c_{n}\right)$ is a constant value and $h\left(X_{i}, \mathbf{c}_{-i}\right)$ represents the function value for the case that all inputs except $X_{i}$ are fixed at their respective cut point coordinates. MDRM is particularly useful for approximating the integrals required for evaluation of the sensitivity indices described in the previous section.

In order to write compact mathematical expression, $\rho_{k}$ and $\theta_{k}$ denoted as the $k$ mean and mean square values, respectively, associate with input parameters $X_{k}$. The Gaussian quadrature is computationally efficient for one-dimensional integrals calculation. The definitions are as follows:

$$
\begin{aligned}
\rho_{k} & =E\left[h_{k}\left(X_{k}\right)\right] \\
& \approx \sum_{l=1}^{N} w_{k l} h\left(c_{1}, \ldots, c_{k-1}, X_{k l}, c_{k+1}, \ldots, c_{n}\right), \\
\theta_{\mathrm{k}} & =E\left[\left[h_{k}\left(X_{k}\right)\right]^{2}\right] \\
& \approx \sum_{l=1}^{N} w_{k l}\left[h\left(c_{1}, \ldots, c_{k-1}, X_{k l}, c_{k+1}, \ldots, c_{n}\right)\right]^{2},
\end{aligned}
$$

where $N$ is the total number of integration points and $X_{k l}$ and $w_{k l}$ are the $l$ th Gaussian integration abscissa and weight function, respectively. The total number of function evaluations required to calculate the sensitivity indices using this approach is $N_{\mathbf{X}} \times N$ with $N_{\mathbf{X}}$ as number of design parameters. 
For computed $\theta_{i}$ and $\rho_{i}(i=\{1, \ldots, n\})$, the primary and total sensitivity indices are approximated as shown as follows:

$$
\begin{aligned}
S_{i} & =\frac{E_{i}\left(V\left(\mathrm{OF} \mid X_{i}\right)\right)}{V(\mathrm{OF}(\mathbf{X}))} \approx \frac{\theta_{i} / \rho_{i}^{2}-1}{\prod_{k=1}^{n} \theta_{k} / \rho_{k}^{2}-1}, \\
S_{T_{i}} & =1-\frac{E_{i}\left(V\left(\mathrm{OF} \mid \sim X_{i}\right)\right)}{V(\mathrm{OF}(\mathbf{X}))} \approx \frac{1-\rho_{i}^{2} / \theta_{i}}{1-\prod_{k=1}^{n} \rho_{k}^{2} / \theta_{k}} .
\end{aligned}
$$

The primary sensitivity index, $S_{i}$, gives an indication of how strong the direct influence of $X_{i}$ is on OF without any interactions with other variables. The total sensitivity index, $S_{T_{i}}$, gives an indication of the total influence of $X_{i}$ on OF from its own direct effects along with its interaction with other variables $[49,50]$.
The differences between the primary effect and the total effect then give an indication of how important the interactions of $X_{i}$ are with other variables in influencing OF. If the main effect is small, whereas the total effect is large, then $X_{i}$ does influence OF but only through interactive effects with other system inputs.

The introduced sensitivity indices accuracy depends on number of integration points used that can be determined via a convergence study $[24,51]$.

4.2. System Input Parameters Used in GSA. Assuming the bending model with torsional parameters imposed to the system, we will have a model which contains bending and torsional response of the system. In order to apply GSA for this model, we propose the following set of input parameters as follows:

$$
\mathbf{X}_{\mathrm{TOT}}=\left[\begin{array}{llllllllllllllllllllllllllll}
J_{r} & J_{g} & I_{r} & m_{D} & m_{e} & l & l_{b} & k_{\phi} & k_{\psi} & k_{b} & k_{b}^{\psi} & C_{\phi} & C_{\psi} & C_{b} & C_{b f} & E I & \alpha_{b} & M_{\text {rip }}^{(1)} & M_{\text {rip }}^{(2)} & M_{\text {rip }}^{(3)} & \mathrm{BL} & M_{\mathrm{BH}}^{\phi_{r}}
\end{array}\right] .
$$

The nominal values of the input parameters as well as the range of parameters variation are given in Table $1 . X^{\star}$ is chosen as cut point (see Section 4.1.1).

\section{Results from GSA of High Speed Shaft (HSS) Model}

In this section, the global sensitivity analysis of the developed mathematical models corresponding to high speed shaft drive train system has been presented. Both torsional and bending models are examined under three different operational scenarios with different motor speeds in steady state $\left(\mathrm{OS}_{i}=\right.$ $\left.\omega_{g}^{\max }=\left[\begin{array}{lll}50 & 40 & 30\end{array}\right] \mathrm{Hz}\right)$.

The analysis presented here focuses on the response characteristics particular to chosen objective functions. It is expected that the operational scenarios will influence the dynamics and structural response to different input variables.

5.0.1 Convergence Study. The number of function evaluations using M-DRM method is $N_{\mathbf{X}} \times N$. For specific set of input variables and on each operational scenario, all objective functions are evaluated at the same time by a single time integration of the model. In order to achieve an acceptable approximation of the sensitivity analysis indices using the M-DRM method, it is important to assess the convergence of the results by increasing number of Gaussian quadrature integration abscissas denoted by $N$ in (18).

The convergence study shows that for a nonlinear system such as high speed shaft drive train with system input parameters prescribed in Section 5, sufficient level of accuracy for the sensitivity analysis can be obtained with $21 \times 20=420$ $\left(N_{\mathbf{X}}=21\right.$ design parameters and $N=20$ integration abscissas) function evaluations. This is one of the most prominent aspects of the global sensitivity analysis using this approach, which could reduce the computational effort drastically.

5.1. GSA of Drive Train High Speed (HSS) Shaft Subsystem Model. In this section we apply GSA to the total model which comprises bending and torsional parameters all together. The input parameters are defined in (20).

For the model containing all bending and torsional parameters, the primary and total sensitivity indices are as shown in Figure 11.

It can be concluded from this that the torsional input parameters (coupling torsional stiffness $k_{\phi}$ and bearing damping due to friction $C_{b f}$ and motor torque ripples $M_{\text {rip }}^{(i)}$, backlash parameter $\mathrm{BL}$, and bearing impact disturbance $M_{\mathrm{BH}}^{\phi_{r}}$ ) do not have significant influence to the bending objective functions $\left(\mathrm{OF}_{B}^{1-11}\right)$. The same conclusion could be made based on the bending input parameters (coupling bending stiffness $k_{\psi}$ and bearing mounting stiffness $k_{b}$ and shaft bending stiffness $E I$ ) which do not seem to have major effect on torsional objective function $\left(\mathrm{OF}_{T}^{2}\right)$.

Based on this analysis, we conclude that the torsional and bending response of the model can be analysed separately, which allows for substantially simpler analysis, which will discussed next.

5.2. HSS Bending Model. After separating the two models, we analyse the sensitivity of HSS within the frame of bending model based on bending objective functions such as deflection field in bearing hub and tip in both lateral and vertical directions and the corresponding frequency domains.

Considering the separation of model, the simulation results of the bending model have been obtained based on semi-inverse dynamics approach ("perfect" speed control), by prescribing the motor angular speed, where it constitutes 


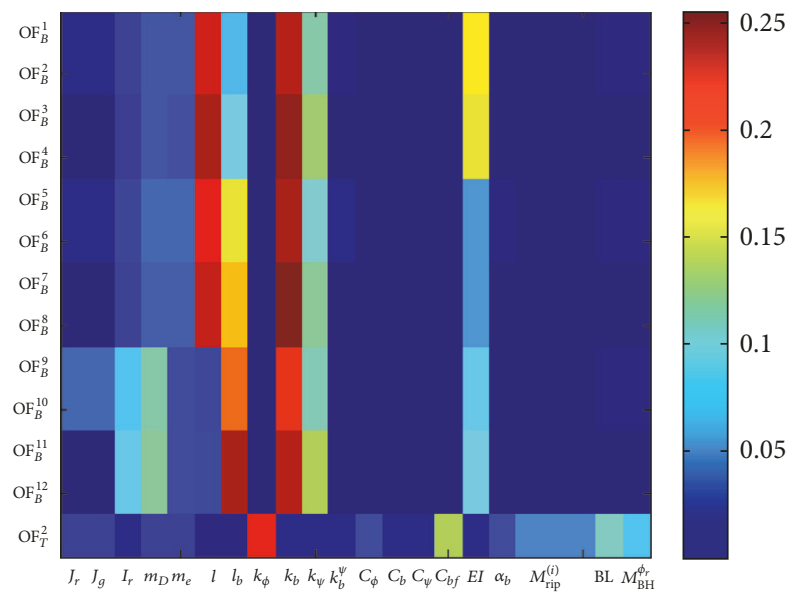

(a)

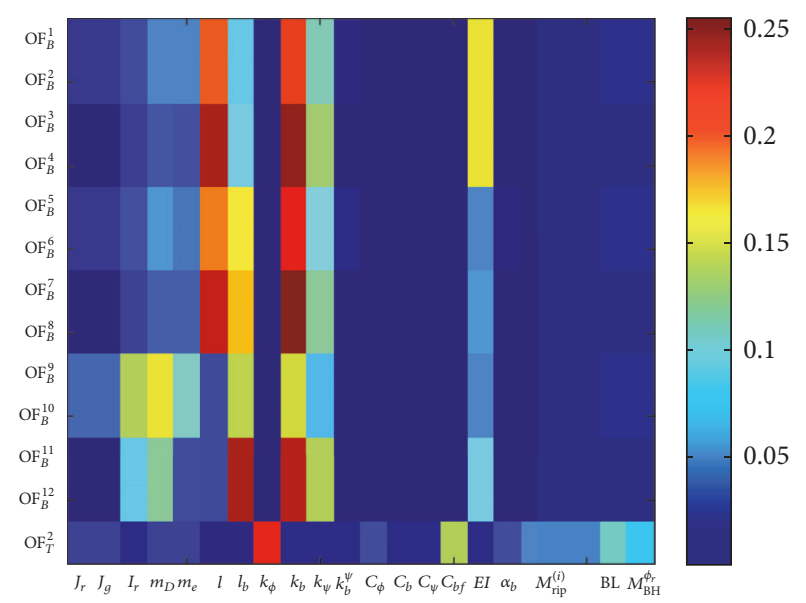

(b)

FIgURE 11: Primary sensitivity $S_{i}(\mathrm{a})$ and total sensitivity $\mathrm{ST}_{i}(\mathrm{~b})$ indices for combined bending and torsional model for $\mathrm{OS}_{1}$.

the input to the system and is prescribed as a ramp loading expressed by Heaviside function:

$$
\begin{aligned}
\omega_{g}(t)= & \frac{\omega_{g}^{\max }}{t_{0}} t H\left(t_{0}-t\right)+\omega_{g}^{\max } H\left(t-t_{0}\right) H\left(t_{1}-t\right) \\
& +\omega_{g}^{\max }\left(\frac{t_{\text {end }}-t}{t_{\text {end }}-t_{1}}\right) H\left(t-t_{1}\right),
\end{aligned}
$$

where $t_{0}$ and $t_{1}$ denote the instant of time of the end of transient and instant of time of the start of the shutdown operations, respectively. $t_{\text {end }}$ is duration of the operational scenarios (see Figure 6).

In order to investigate the bending model sensitivity, the input parameter appearing in the governing equations corresponding to the model is chosen as follows:

$$
\mathbf{X}_{B}=\left[\begin{array}{lllllllllllllllllll}
J_{r} & J_{g} & I_{r} & m_{D} & m_{e} & l & l_{b} & k_{\phi} & k_{\psi} & k_{b} & k_{b}^{\psi} & C_{\phi} & C_{\psi} & C_{b} & C_{b f} & E I & \alpha_{b} & M_{\mathrm{BH}}^{\psi}
\end{array}\right] .
$$

Here, the sensitivity analysis has been performed for bending model for 3 different operational scenarios with different motor speeds in steady state regime.

It was shown that within these 3 different motor frequencies, the contribution of the input parameters is not changing drastically. The obtained results of GSA are presented in Figure 12. The results indicates that the most influential structural parameters which have the major effect to the objective functions are coupling bending stiffness $k_{\psi}$ and bearing mounting stiffness $k_{b}$, shaft bending stiffness $E I$, and bearing hub and tip locations $l_{b}, l$. Also, the damping parameters do not have significant effect on both primary and total sensitivity indices.

More detailed analysis of the results based on each objective functions and influence of the input parameters is presented as follows.

(i) Tip Deflection in Vertical and Lateral Directions in Steady State $\left(\mathrm{OF}_{B}^{1-2}\right)$ and Shutdown Scenarios $\left(\mathrm{OF}_{B}^{3-4}\right)$. It is illustrated that the coupling bending stiffness $k_{\psi}$ and bearing hub mounting stiffness $k_{b}$ are the most dominant influential parameters, respectively.

It could be seen from Figure 12 that the shaft bending stiffness $E I$ has more influence in tip deflection field compared to the other objective function. This is somehow logical since the first bending mode shape is higher in tip $(x=l)$ compared to bearing hub $\left(x=l_{b}\right)$ [33].

Moreover, the geometric locations of bearing hub and tip $\left(l_{b}, l\right)$ are influential parameters in terms of deflection field in both bearing hub and tip.

(ii) Bearing Hub Deflection in Vertical and Lateral Directions in Steady State $\left(\mathrm{OF}_{B}^{5-6}\right)$ and Shutdown Scenarios $\left(\mathrm{OF}_{B}^{7-8}\right)$. In bearing deflection field the influence of $l_{b}$ is more than $l$. In tip deflection field the influence of $l$ is more than $l_{b}$.

The bearing bending stiffness $k_{b}^{\psi}$ does not have a major effect to the system objective functions.

It could be seen that the angle dependency of the bearing stiffness $\alpha_{b}$ is not having a major effect to all of objective functions, since the realistic range for $\alpha_{b}$ is small.

(iii) Frequency Response of the Tip Deflection in Vertical and Lateral Directions in Steady State $\left(O F_{B}^{9-10}\right)$ and Shutdown Scenarios $\left(O F_{B}^{11-12}\right)$. The influences of stiffness parameters $k_{\psi}$ and $k_{b}$ and $E I$ and $l_{b}$ and also inertia parameters $I_{r}, m_{D}$, and $m_{e}$ are the most dominant parameters, respectively. This tells that aforementioned parameters are the most important ones in terms of frequency response of the tip deflection. 


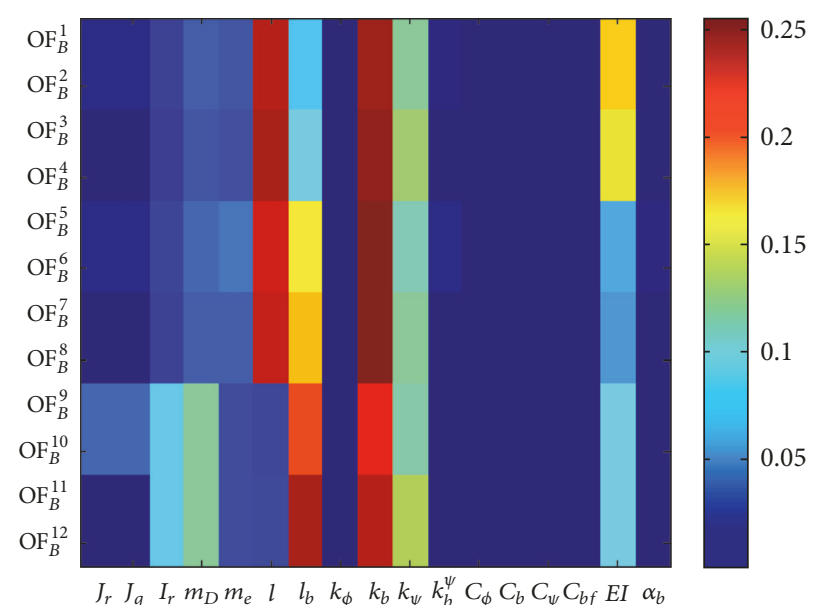

(a)

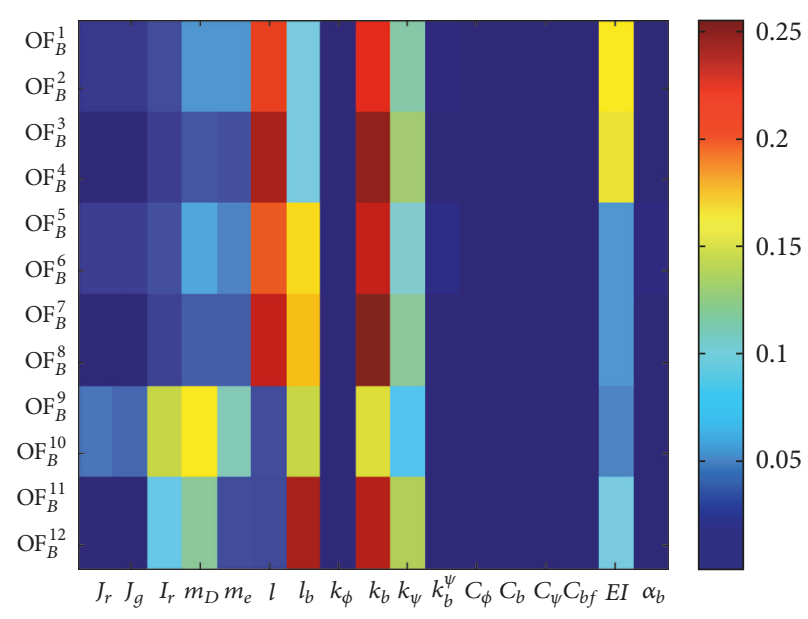

(b)

FIGURE 12: Primary sensitivity $S_{i}(\mathrm{a})$ and total sensitivity $\mathrm{ST}_{i}(\mathrm{~b})$ indices for bending model for $\mathrm{OS}_{1,2,3}$.

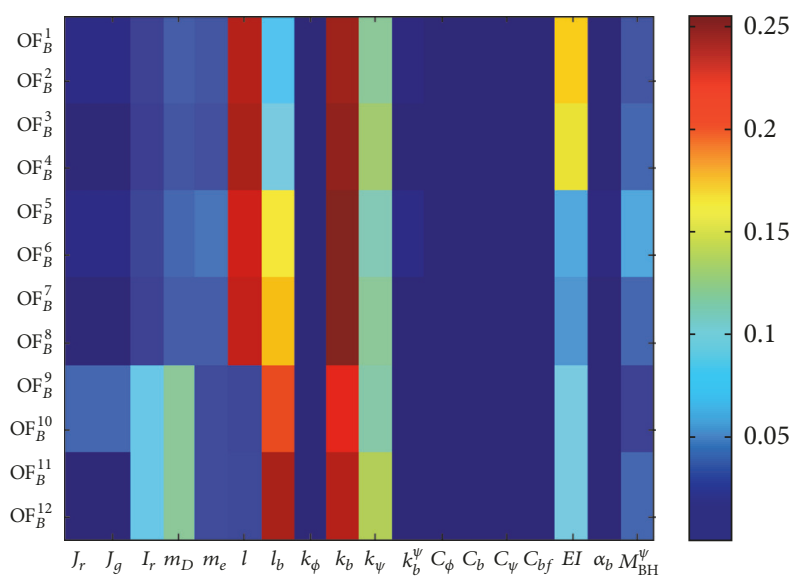

(a)

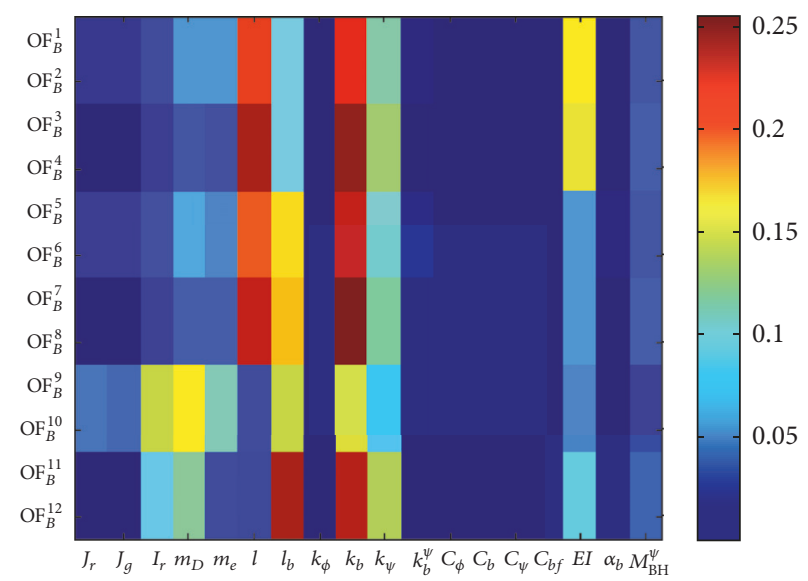

(b)

FIgURE 13: Primary sensitivity $S_{i}$ (a) and total sensitivity $\mathrm{ST}_{i}(\mathrm{~b})$ indices for bending model with bearing defect assumption for $\mathrm{OS}_{1}$.

Among the mass inertia parameters, $I_{r}, m_{e}$, and $m_{D}$ affect most of the bending objective functions.

It could be also seen form Figure 12 that the damping input parameters have the least influences on both primary and total sensitivity indices.

To sum up the conclusion related to the GSA of HSS subsystem within the frame of bending model, $k_{b}$ and $k_{\psi}$ and $E I$ and $I_{r}$ and $m_{D}$ and $m_{e}$ have the major effects on both primary and total sensitivity indices.

However, according to the sensitivity analysis results, rest of parameters stated in the system modelling do not have a remarkable effect on the mentioned objective functions. Such information would be particularly useful in attenuating the number of design parameters for optimization applications of wind turbine drive trains setups which leads to cost efficient simulations.

5.3. Bending Model with Bearing Defect: Detection of Faults in Inner Ring. Here, we study the bearing defect influence to the objective functions. The aim is to investigate the sensitivity indices with respect to the bearing defect parameter $M_{\mathrm{BH}}^{\psi}$ imposing a radial load to the bearing housing and seek its effect to the bearing deflection and loads.

It is hypothesized that if the sensitivity index with respect to parameter $M_{\mathrm{BH}}^{\psi}$ is comparable to other sensitivities in certain $\mathrm{OF}$, we can expect that a sensor measuring this $\mathrm{OF}$ is able to detect defects of size related to the magnitude of $M_{\mathrm{BH}}^{\psi}$. The obtained results on GSA with bearing defect are presented in Figure 13 and summarized below.

The minimum value of $M_{\mathrm{BH}}^{\psi}$ which makes the sensitivity indices to be detectable has been investigated.

(i) Deflection fields in vertical and lateral directions in steady state and shutdown scenarios for the tip $\left(\mathrm{OF}_{B}^{1-4}\right)$ and bearing hub $\left(\mathrm{OF}_{B}^{5-8}\right)$, respectively: in Figure 13, comparing $M_{\mathrm{BH}}^{\psi}$ sensitivity indices for objective functions tip and bearing hub deflections $\left(\mathrm{OF}_{B}^{1-4}\right.$ versus $\left.\mathrm{OF}_{B}^{5-8}\right)$, the influence of the bearing 
defect $M_{\mathrm{BH}}^{\psi}$ in bearing hub is more than the tip. This is due to direct influence of the defect in the bearing by imposing the defect torque, which means that the bearing may experience more damage by introducing a defect in the inner ring.

(ii) Frequency response of the tip deflection in steady state $\left(\mathrm{OF}_{B}^{9-10}\right)$ and shutdown $\left(\mathrm{OF}_{B}^{11-12}\right)$ scenarios: in FFT of tip deflection, bearing defect has shown different influence compared to the healthy case (no defect). This means that the frequency response of structure in both healthy and faulty conditions is different and by studying FFT of system response and investigating their sensitivity indices, we could detect some faults sources in specific components.

5.4. HSS Torsional Model. The torsional model has been studied based on the faults sources introduced in the previous sections. Backlash effects on coupling creating nonlinearities to the system structure, motor torque ripple with specific peaks in the frequency domain, and bearing defect disturbance torque imposing on the rotor angular direction are applied within system modelling. In order to optimize the design and upgrade the structural performance, the system response must be studied based on these assumptions and compared with the healthy conditions. This gives a better insight to understand the faults sources and could facilitate the way to prevent the failures in drive train by creating structures which are less sensitive with respect to these faults sources. This could be studied within GSA by investigating and optimizing the sensitivity indices.

The sensitivity analysis for torsional model has been performed based on 3 different operational scenarios (see Figure 6) for motor speeds in steady state regime. In order to investigate the torsional model sensitivity analysis, the input parameters appearing in the governing equations corresponding to the model are chosen as follows:

$$
\begin{aligned}
& \mathbf{X}_{T} \\
& =\left[\begin{array}{lllllllllllll}
J_{r} & J_{g} & m_{e} & k_{\phi} & C_{b r}^{f} & C_{b g} & M_{\text {rip }}^{(1)} & M_{\text {rip }}^{(2)} & M_{\text {rip }}^{(3)} & \text { BL } & M_{\mathrm{BH}}^{\phi_{r}}
\end{array}\right] .
\end{aligned}
$$

5.4.1. Torsion Vibration Model of Drive Train Test Rig with Backlash. Upon considering only the torsional response of the test rig, the complete model in Section 2.1 is reduced, a lumped torsion vibration model. An engineering abstract of the model is depicted in Figure 14 and entails two inertias, disk $J_{r}$ and generator rotor $J_{g}$, respectively. Each of these inertias is supported by bearings. The shaft and coupling flexibility is lumped together and represented by a torsion spring. Due to gravity and eccentric mass, there will be an external torque $M_{r}\left(=m_{e} r_{e} g \cos \phi_{r}\right)$ acting on the disk, and the induced electrical torque from the motor $M_{g}$ acts on the generator rotor.

Hence, we have the following set of equations to describe the test rig torsional vibration response:

$$
\begin{aligned}
{\left[J_{r}+m_{e} r_{e}^{2}\right] \ddot{\phi}_{r}+C_{b r}^{f} \dot{\phi}_{r}+k_{\phi}\left(\phi_{r}-\phi_{g}\right) } & =M_{r}(t), \\
J_{g} \ddot{\phi}_{g}+C_{b g} \dot{\phi}_{g}+k_{\phi}\left(\phi_{g}-\phi_{r}\right) & =M_{g}(t) .
\end{aligned}
$$

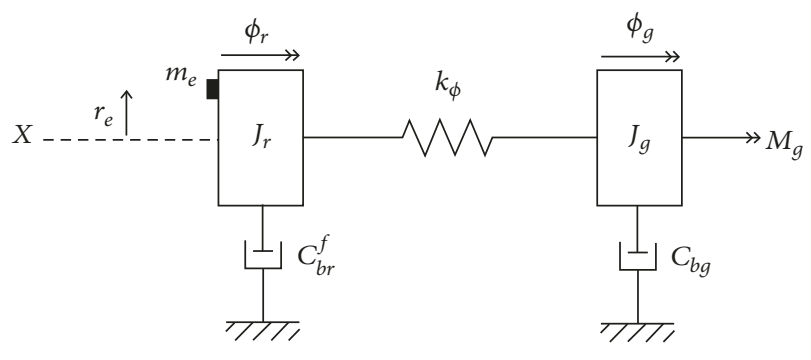

FIGURE 14: Engineering abstract of the test rig in torsional vibration.

Here, we define $C_{b r}^{f}\left(=C_{b r}+C_{b f}\right)$ as combination structural damping in rotor and the bearing damping due to the friction.

Based on the results (see Figure 15), it could be demonstrated that the most influential input parameters to the torsional objective functions are coupling torsional stiffness $k_{\phi}$ and backlash in coupling BL, motor torque ripples $M_{\text {rip }}^{(i)}$, and bearing defect parameter $M_{\mathrm{BH}}^{\phi_{r}}$. Also, the structural damping of the rotor and bearing friction coefficient $C_{b r}^{f}$ has some effects to both primary and total sensitivity indices. The effects of damping coefficient (except the bearing damping) and rotor and motor mass inertia values $\left(J_{r}\right.$ and $\left.J_{g}\right)$ are not significant compared to the other aforementioned parameters. In total sensitivity $S_{T_{i}}$, also eccentric mass has shown some impact which is expectable since it imposes an external torque to the structure.

More detailed analysis of the results based on each objective functions and influence of the input parameters is stated as follows.

(i) Coupling Torque in Transient and Steady State Regimes $\left(O F_{T}^{1-2}\right)$. The coupling torque has strong sensitivity in terms of torque ripple parameters $M_{\text {rip }}^{(i)}(i=\{1,2,3\})$ arising from the motor. This tells the significance of motor ripple assumption in drive trains as one type of fault source.

(ii) Coupling Torque and Its FFT in Shutdown Regime $\left(\mathrm{OF}_{T}^{3,6}\right)$. It is clear that in shutdown regime there is no any torque ripple imposed by the motor, of which objective functions $\mathrm{OF}_{T}^{3}$ and $\mathrm{OF}_{T}^{6}$ sensitivity indices for torque ripples are identically zero.

In FFT, coupling torque influence is more than steady state case, since there is no ripple imposed by the motor. Also eccentric mass has some contribution especially in total sensitivity index.

(iii) Frequency Response of the Coupling Torque in Steady State Regime $\left(\mathrm{OF}_{T}^{4-5}\right)$. In FFT of the coupling torque in steady state regime, the most dominant parameter is $k_{\phi}$. Then the backlash and motor torque ripples have significant contribution. This tells that in vibration analysis it is very important to identify the vibration faults sources namely here backlash and ripple, which may affect the system structure in a severe way. 


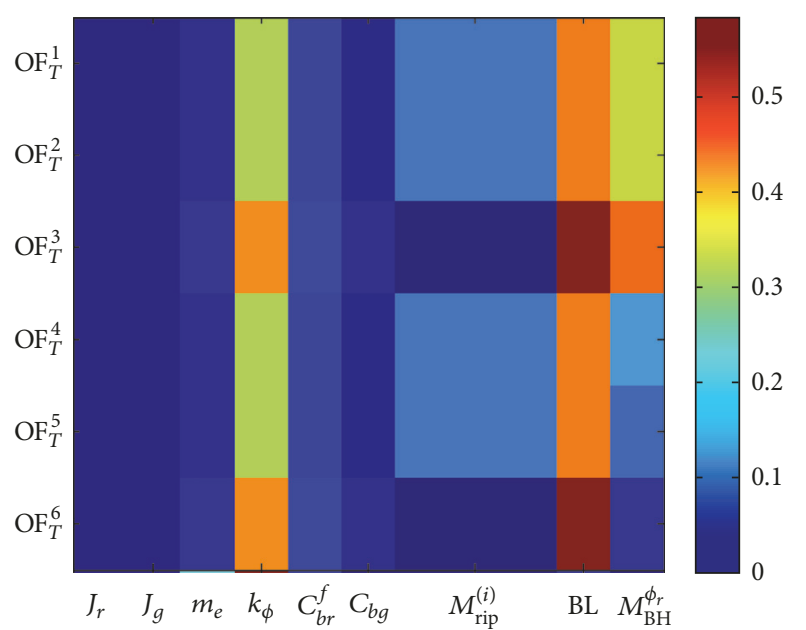

(a)

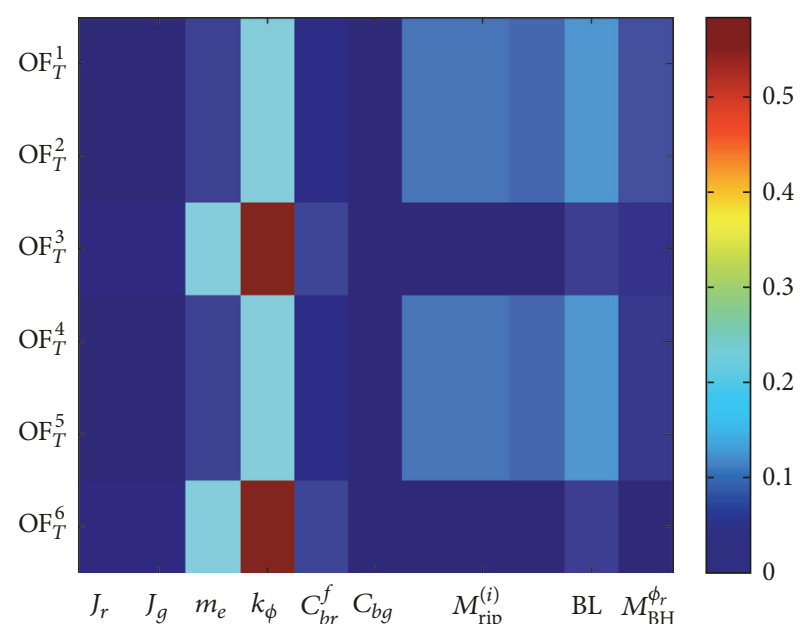

(b)

FIGURE 15: Primary sensitivity $S_{i}(\mathrm{a})$ and total sensitivity $\mathrm{ST}_{i}(\mathrm{~b})$ indices for torsional model for $\mathrm{OS}_{1}$ with backlash.

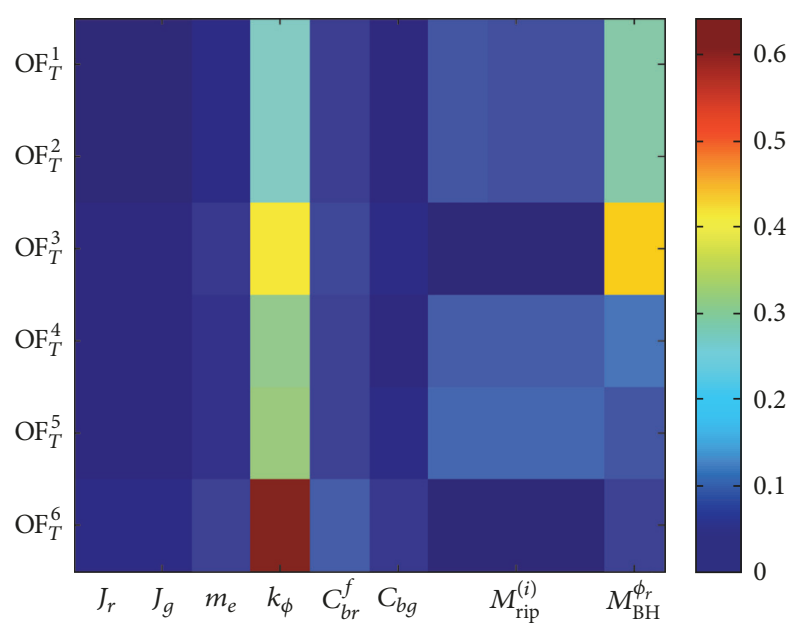

(a)

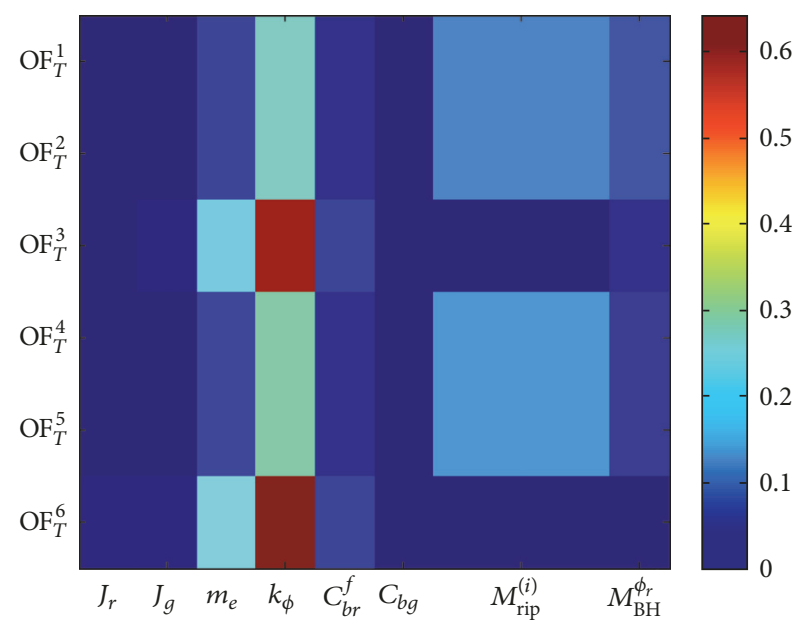

(b)

FIGURE 16: Primary sensitivity $S_{i}$ (a) and total sensitivity $\mathrm{ST}_{i}(\mathrm{~b})$ indices for torsional model for $\mathrm{OS}_{1}$ with no backlash.

(iv) Bearing Defect Maximum Value $M_{B H}^{\phi_{r}}$. By increasing the bearing defect maximum torque (Figure 5), we could investigate its contribution to the sensitivity indices. The aim is to define the minimum value of defect parameter $M_{\mathrm{BH}}^{\max }$ such that the sensitivity indices related to this parameter would be larger than a specific value $\left(M_{\mathrm{BH}}^{\max }>100 \mathrm{Nm}\right)$. This methodology contributes to detect, predict, and prevent the faults and failures in bearings.

Summing up the results of GSA for torsional model, we could state that the most influential parameters are $k_{\phi}$ and faults sources BL and $M_{\text {rip }}^{(i)}$ and also $C_{b f}$, respectively.

For disturbance torque coming from bearing housing $M_{\mathrm{BH}}^{\phi_{r}}$, we notice that its influence is more in total sensitivity index rather than primary one. This means that $M_{\text {rip }}^{(i)}$ influence is more when the other parameters are changed. The same conclusion could be made for ripple parameters $M_{\text {rip }}^{(i)}$.
5.4.2. Torsional Model with No Backlash. In order to emphasize into the bearing fault detection, we study the system structure response based on no backlash (Figure 16). Then, we investigate the influence of the input parameters to the objective functions in case when the shaft coupling has a backlash.

The obtained results have led to the following conclusions:

(i) The bearing defect (quantified in terms of $M_{\mathrm{BH}}^{\phi_{r}}$ ) could be detected easier in case where no backlash compared to with backlash (more contribution sensitivity indices especially the primary one). This is very important conclusion and means that, in the systems with a couple of sources of faults, the detectability of each fault may not be easy and it get more complex by introducing more complex system and assuming more faults. 


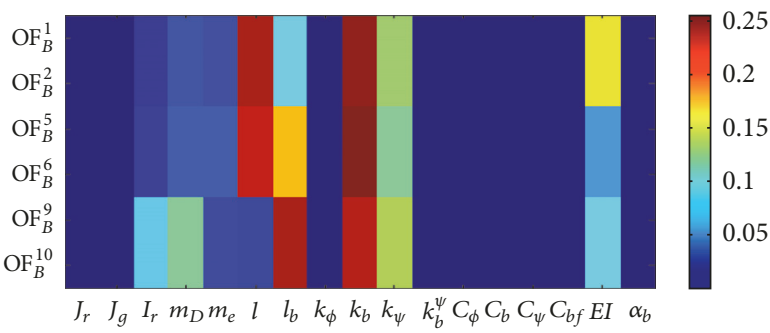

(a)

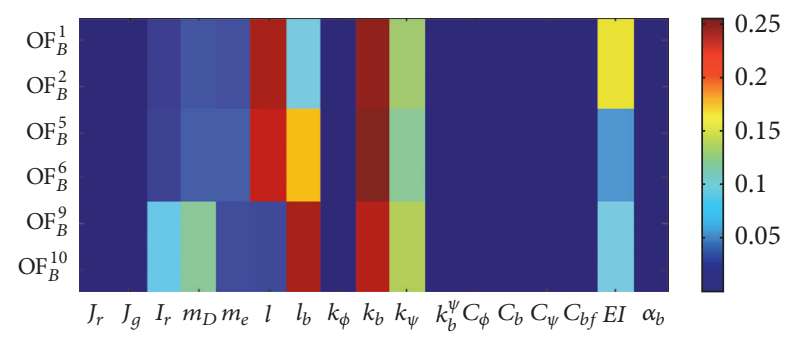

(b)

FIgURe 17: Primary sensitivity $S_{i}$ (a) and total sensitivity $\mathrm{ST}_{i}$ (b) indices for bending model for $h_{\text {res }}$.

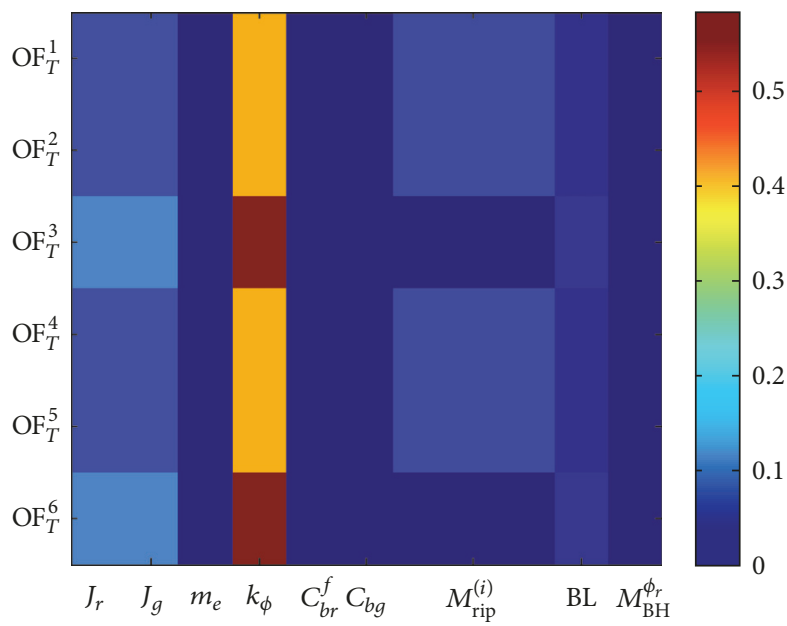

(a)

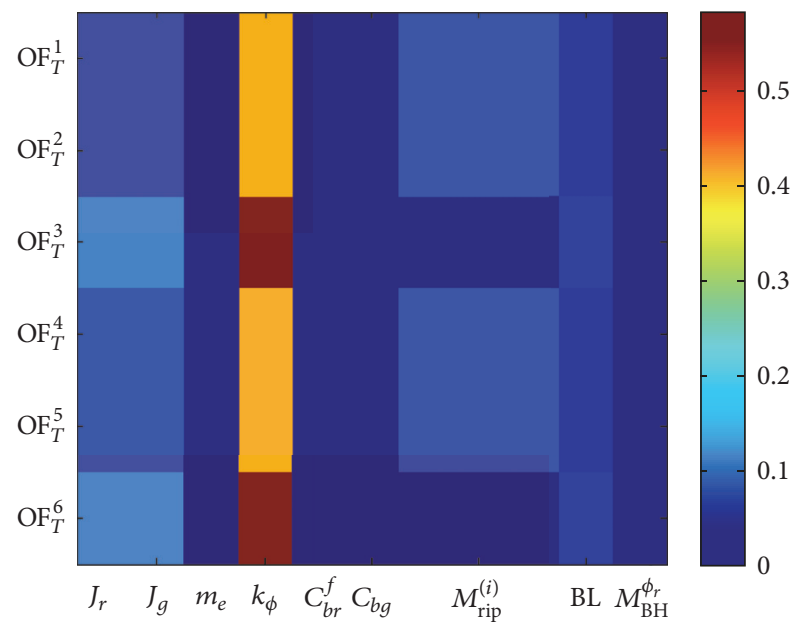

(b)

FIgURE 18: Primary sensitivity $S_{i}(\mathrm{a})$ and total sensitivity $\mathrm{ST}_{i}(\mathrm{~b})$ indices for torsional model for $h_{\text {res }}$.

(ii) The influence of bearing defect in the FFT for first peak and the second peaks is small, though recognizable. This draws the same conclusion for the bending model with defect, which tells that some specific peaks in the FFT correspond to the system structural faults.

5.5. Special Cases: Resonance Investigation. The operating speed ranges in wind turbines and system excitation frequency have a wide range. The system may encounter resonances leading to severe vibration and affect the normal operation of the machine. Thus, the drive train dynamics must be studied with more focus in excitation ranges.

To focus on the excitation ranges, we investigate the primary and secondary sensitivity indices within the case in which the input parameters leads to resonance. It means that we only take the randomized input parameters which are near to the resonance frequency of the system in terms of bending and torsion:

$$
\begin{aligned}
\mathbf{X}_{\text {res }} & =\{\mathbf{X} \text { such that norm }(\operatorname{eig}(\operatorname{SYS}(\mathbf{X})) \\
& -\operatorname{eig}(\operatorname{SYS}(\mathbf{c}))) \leq \text { TOL }\},
\end{aligned}
$$

where SYS $(\mathbf{X})$ and SYS(c) denote for the system eigenvalue based on randomized set of $\mathbf{X}$ and nominal structural parameters presented in Table 1, respectively. Also TOL = $1 \mathrm{~Hz}$ is considered as a tolerance for frequency fluctuation.

Some conclusions could be made based on aforementioned cases, and the results are presented in Figures 17 and 18.

(i) By selecting those randomized variables which based on them the eigenfrequency of the structure is near to specific value $(50 \mathrm{~Hz})$, the sensitivity of eigenfrequency variables takes the most contribution. This is expectable since in the resonance points the system influential parameters are only the ones which the eigenfrequency of the system is based on them.

(ii) In optimum case, where the randomized values are identical to the bending and torsional eigenfrequencies, we expect that only the input parameters have influence that they are in the eigenfrequency definition.

\section{Conclusion and Outlook}

The global sensitivity analysis for both torsional and bending models of high speed shaft drive train test rig with respect to its structural parameters has been carried out using M-DRM method. 
The current study showed the feasibility and efficiency of the M-DRM for global sensitivity analysis of nonlinear dynamical system (high speed shaft drive train), which provided the results in a computationally efficient framework. The M-DRM applied here revealed practical significant results which can reduce the number of input design parameters for optimization of wind turbine drive train systems.

By comparing the sensitivity of objective functions with respect to different input structural parameters, a better understanding of drive train system dynamics is obtained. Vertical and lateral deflections in bearing housing and tip, coupling torque in 3 different operational scenarios, FFT of these functions, have been introduced as the objective functions to represent the test rig dynamic performance. To reflect the effect of operational scenarios, the aforementioned outputs have been evaluated within transient, steady state, and shutdown cases in 3 different motor speeds. The effect of the fundamental structural parameters on the dynamic response of the HSS drive train test rig has been recognized. By comparing the sensitivity indices of corresponding objective functions, a better understanding of drive train system dynamics is obtained.

Based on the present analysis, the following conclusions could be stated:

(i) GSA for complex systems is computationally expensive by using ordinary algorithms like Monte Carlo. Nevertheless, M-DRM method has been successfully applied to high speed shaft drive train model and provided physically reasonable results in an efficient computational framework.

(ii) For considered HSS drive train system model, the estimated primary and total sensitivity indices coincide, revealing no variance interaction effects between the input parameters within different operational scenarios. Also there is a direct correspondence between $S_{i}$ and $S_{T_{i}}$, which means that the higher order effects are negligible.

(iii) Deflection fields in both tip and bearing hub are mostly sensitive with respect to bearing mounting stiffness $k_{b}$ and coupling bending stiffness $k_{\psi}$ and geometric parameters $l, l_{b}$ and shaft bending stiffness EI.

(iv) Coupling torque mostly in all regimes is influenced mainly by coupling torsional stiffness $k_{\phi}$, coupling backlash $\mathrm{BL}$, and motor torque ripples $M_{\text {rip }}^{(i)}$, respectively. This means that the proposed faults sources may lead to drastic contributions in components loads. In this research we have quantified these contributions.

(v) Dynamic modelling and vibration responses simulation are important for fault mechanism studies to provide proofs for defect detection and fault diagnose. The most significant demonstration in the presented research is the applicability of GSA towards defect detection and faults diagnose in functional components of drive train. The results suggest that, from sensitivity indices, detectability of some faults such as bearing defect could be analysed. This analysis illustrates the applicability of the GSA method for advancing modern condition monitoring systems for bearings.

The global sensitivity analysis provided a useful method to deeply understand some aspects of the initial structural parameters to the dynamic outputs. At this stage, a preliminary assessment of the key parameters has been performed. Moreover, fault modelling in functional components such as motor ripples, bearing defects, coupling backlash, and their detectability within GSA has been investigated. This facilitates understanding the faults sources and their effect to other functional components, which leads to better design and optimize drive trains more efficiently with less failure.

Finally, the items for future work could be outlined as follows:

(i) Apply GSA for real wind turbine structural parameters such that we first propose to apply the same analysis for NREL $5 \mathrm{MW}$ wind turbine and investigate GSA sensitivity indices.

(ii) Carry out dimensional analysis and upscaling of the test rig and apply GSA for Pi Buckingham theory: the aim is to prescribe system structure based on minimal number of dimensionless parameters, which could lead to upscale the structure and predict the real wind turbine behaviours.

(iii) Apply wind turbine aerodynamic loads as an external load to the rotor $\left(M_{r}\right)$ : in order to get more realistic response of the system, considering the stochastic nature of wind loads, it would be interesting to study the current model based on realistic calculated aerodynamic loads extracted from FAST software.

(iv) Study the bearing modelling in more advanced and detailed approach: the bearing defects here have been modelled by introducing an radial excitation to certain DOFs imposed on inner ring of the bearing. For future work, it is important to consider also ball mass inertia and outer ring dynamics $[40,41]$.

(v) Model the electromechanical interaction of wind turbine drive train: since wind turbine contains both mechanical and electrical components, to have better understanding of drive train dynamics, the study must be done within this interaction.

\section{Conflicts of Interest}

The authors declare that they have no conflicts of interest.

\section{Acknowledgments}

This project is financed through the Swedish Wind Power Technology Centre (SWPTC). SWPTC is a research centre for design of wind turbines. The purpose of the centre is to support Swedish industry with knowledge of design techniques as well as maintenance in the field of wind power. 
The centre is funded by the Swedish Energy Agency and Chalmers University of Technology as well as academic and industrial partners. In particular, a donation of motors and frequency converters by $\mathrm{ABB} \mathrm{CR}$, WindCon 3.0 condition monitoring system, and set of sensors by SKF is gratefully acknowledged. Besides, the authors would like to thank Jan Möller for his contribution in the laboratory.

\section{References}

[1] International Renewable Energy Agency, Wind Power, vol. 1, Power sector, Issue 5/5 of Renewable energy technologies: Cost analysis series.

[2] F. Dincer, "The analysis on wind energy electricity generation status, potential and policies in the world," Renewable \& Sustainable Energy Reviews, vol. 15, no. 9, pp. 5135-5142, 2011.

[3] J. Ribrant and L. Bertling, "Survey of failures in wind power systems with focus on Swedish wind power plants during 19972005," in Proceedings of the 2007 IEEE Power Engineering Society General Meeting, PES, USA, June 2007.

[4] J. L. M. Peeters, D. Vandepitte, and P. Sas, "Analysis of internal drive train dynamics in a wind turbine," Wind Energy, vol. 9, no. 1-2, pp. 141-161, 2006.

[5] F. Oyague, "Gearbox Modeling and Load Simulation of a Baseline 750-kW Wind Turbine Using State-of-the-Art Simulation Codes," Tech. Rep. NREL/41160, 2009.

[6] W. Shi, H.-C. Park, S. Na, J. Song, S. Ma, and C.-W. Kim, "Dynamic analysis of three-dimensional drivetrain system of wind turbine," International Journal of Precision Engineering and Manufacturing, vol. 15, no. 7, pp. 1351-1357, 2014.

[7] S. Asadi, Drive train system dynamic analysis: application to wind turbines, Licentiate Thesis, Chalmers University of Technology, 2016.

[8] F. Oyague, Fault Identification in Drive Train Components Using Vibration Signature Analysis, TP-500-41160, National Renewable Energy Laboratory, 2009.

[9] K. G. Scott, D. Infield, N. Barltrop, J. Coultate, and A. Shahaj, "Effects of extreme and transient loads on wind turbine drive trains," in Proceedings of the 50th AIAA Aerospace Sciences Meeting Including the New Horizons Forum and Aerospace Exposition, January 2012.

[10] G. Semrau, S. Rimkus, and T. Das, "Nonlinear Systems Analysis and Control of Variable Speed Wind Turbines for Multiregime Operation," Journal of Dynamic Systems, Measurement, and Control, vol. 137, no. 4, Article ID 041007, 2015.

[11] T. M. Ericson and R. G. Parker, "Natural frequency clusters in planetary gear vibration," Journal of Vibration and Acoustics, vol. 135, no. 6, Article ID 061002, 2013.

[12] M. Singh and S. Santoso, "Dynamic Models for Wind Turbines and Wind Power Plants," Tech. Rep. NREL/SR-5500-52780, 2011.

[13] J. Wang, D. Qin, and Y. Ding, "Dynamic behavior of wind turbine by a mixed flexible-rigid multi-body model," Journal of System Design and Dynamics, vol. 3, no. 3, pp. 403-419, 2009.

[14] S. Struggl, V. Berbyuk, and H. Johansson, "Review on wind turbines with focus on drive train system dynamics," Wind Energy, vol. 18, no. 4, pp. 567-590, 2015.

[15] C. Zhu, Z. Huang, Q. Tang, and Y. Tan, "Analysis of nonlinear coupling dynamic characteristics of gearbox system about wind-driven generator," Chinese Journal of Mechanical Engineering, vol. 41, no. 8, pp. 203-207, 2005.
[16] B. Iooss and L. Lemaitre, "A review on global sensitivity analysis methods," in Uncertainty management in Simulationoptimization of Complex Systems: Algorithms and Applications, C. Meloni and G. Dellino, Eds., pp. 101-122, Springer US, 2015.

[17] C. I. Reedijk, Sensitivity Analysis of Model Output: Performance of various local and global sensitivity measures on reliability problems, [Master, thesis], Delft University of Technology, 2000.

[18] F. Pianosi, K. Beven, J. Freer et al., "Sensitivity analysis of environmental models: A systematic review with practical workflow," Environmental Modeling and Software, vol. 79, pp. 214-232, 2016.

[19] E. Borgonovo and E. Plischke, "Sensitivity analysis: a review of recent advances," European Journal of Operational Research, vol. 248, no. 3, pp. 869-887, 2016.

[20] X.-Y. Zhang, M. N. Trame, L. J. Lesko, and S. Schmidt, "Sobol sensitivity analysis: A tool to guide the development and evaluation of systems pharmacology models," CPT: Pharmacometrics \& Systems Pharmacology, vol. 4, no. 2, pp. 69-79, 2015.

[21] H. M. Wainwright, S. Finsterle, Y. Jung, Q. Zhou, and J. T. Birkholzer, "Making sense of global sensitivity analyses," Computers \& Geosciences, vol. 65, pp. 84-94, 2014.

[22] X. Zhang and M. D. Pandey, "An effective approximation for variance-based global sensitivity analysis," Reliability Engineering \& System Safety, vol. 121, pp. 164-174, 2014.

[23] A. Saltelli, "Making best use of model evaluations to compute sensitivity indices," Computer Physics Communications, vol. 145, no. 2, pp. 280-297, 2002.

[24] X. Zhang and M. D. Pandey, "Structural reliability analysis based on the concepts of entropy, fractional moment and dimensional reduction method," Structural Safety, vol. 43, pp. 28-40, 2013.

[25] J. M. P. Dias and M. S. Pereira, "Sensitivity Analysis of RigidFlexible Multibody Systems," Multibody System Dynamics, vol. 1, no. 3, pp. 303-322, 1997.

[26] K. D. Bhalerao, M. Poursina, and K. . Anderson, "An efficient direct differentiation approach for sensitivity analysis of flexible multibody systems," Multibody System Dynamics, vol. 23, no. 2, pp. 121-140, 2010.

[27] D. Bestle and J. Seybold, "Sensitivity analysis of constrained multibody systems," Archive of Applied Mechanics, vol. 62, no. 3, pp. 181-190, 1992.

[28] S. M. Mousavi Bideleh and V. Berbyuk, "Global sensitivity analysis of bogie dynamics with respect to suspension components," Multibody System Dynamics, vol. 37, no. 2, pp. 145-174, 2016.

[29] P. M. McKay, R. Carriveau, D. S.-K. Ting, and J. L. Johrendt, "Global sensitivity analysis of wind turbine power output," Wind Energy, vol. 17, no. 7, pp. 983-995, 2014.

[30] K. Dykes, A. Ning, R. King, P. Graf, G. Scott, and P. S. Veers, "Sensitivity Analysis of Wind Plant Performance to Key Turbine Design Parameters: A Systems Engineering Approach," in Conference Paper, NREL/CP-5000-60920, 2014, Contract No. DE-AC36-08GO28308, National Harbor, Maryland, USA, 2014.

[31] J. Helsen, P. Peeters, K. Vanslambrouck, F. Vanhollebeke, and W. Desmet, "The dynamic behavior induced by different wind turbine gearbox suspension methods assessed by means of the flexible multibody technique," Journal of Renewable Energy, vol. 69, pp. 336-346, 2014.

[32] B. Marrant, The validation of MBS multi-megawatt gearbox models on a 13.2 MW test rig, Simpack User Meeting, 2012, 3.

[33] S. Asadi, V. Berbyuk, and H. Johansson, "Vibration Dynamics of a Wind Turbine Drive Train High Speed Subsystem: Modelling 
and Validatio," in Proceedings of the ASME, International Design Engineering Technical Conferences and Computers and Information in Engineering Conference, pp. C2015-46016, August, Boston, Massachusetts, USA, 2015.

[34] S. Asadi, V. Berbyuk, and H. Johansson, "Structural dynamics of a wind turbine drive train high speed subsystem: Mathematical modelling and validation," in Proc. of the International Conference on Engineering Vibration, Ljubljana, 7 - 10 September; [editors Miha Boltezar, Janko Slavic, Marian Wiercigroch] EBook - Ljubljana, pp. 553-562, 2015.

[35] M. Todorov, I. Dobrev, and F. Massouh, "Analysis of torsional oscillation of the drive train in horizontal-axis wind turbine," in Proceedings of the 2009 8th International Symposium on Advanced Electromechanical Motion Systems and Electric Drives Joint Symposium, ELECTROMOTION 2009, France, July 2009.

[36] T. A. Silva and N. M. Maia, "Elastically restrained BernoulliEuler beams applied to rotary machinery modelling," Acta Mechanica Sinica, vol. 27, no. 1, pp. 56-62, 2011.

[37] T. Iwatsubo, Y. Yamamoto, and R. Kawai, "Start-up torsional vibration of rotating machine driven by synchronous motor," in Proc. of the Int. Conference on Rotordynamics, IFToMM.

[38] T. C. Kim, T. E. Rook, and R. Singh, "Effect of smoothening functions on the frequency response of an oscillator with clearance non-linearity," Journal of Sound and Vibration, vol. 263, no. 3, pp. 665-678, 2003.

[39] B. Ghalamchi, J. Sopanen, and A. Mikkola, "Simple and versatile dynamic model of spherical roller bearing," International Journal of Rotating Machinery, vol. 2013, Article ID 567542, 13 pages, 2013.

[40] S. P. Harsha, "Nonlinear dynamic analysis of an unbalanced rotor supported by roller bearing," Chaos, Solitons \& Fractals, vol. 26, no. 1, pp. 47-66, 2005.

[41] L. Niu, H. Cao, Z. He, and Y. Li, "Dynamic modeling and vibration response simulation for high speed rolling ball bearings with localized surface defects in raceways," Journal of Manufacturing Science and Engineering-Transactions of the ASME, vol. 136, no. 4, Article ID 041015, 2014.

[42] Rolling bearings (general catalogue published by SKF group), 2012.

[43] A. Saltelli, P. Annoni, I. Azzini, F. Campolongo, M. Ratto, and S. Tarantola, "Variance based sensitivity analysis of model output. Design and estimator for the total sensitivity index," Computer Physics Communications, vol. 181, no. 2, pp. 259-270, 2010.

[44] A. Saltelli and P. Annoni, "How to avoid a perfunctory sensitivity analysis," Environmental Modeling and Software, vol. 25, no. 12, pp. 1508-1517, 2010.

[45] I. M. Sobol, "Theorems and examples on high dimensional model representation," Reliability Engineering \& System Safety, vol. 79, no. 2, pp. 187-193, 2003.

[46] H. Rabitz and O. F. Alis, "General foundations of highdimensional model representations," Journal of Mathematical Chemistry, vol. 25, no. 2-3, pp. 197-233, 1999.

[47] I. M. Sobol, "Global sensitivity indices for nonlinear mathematical models and their Monte Carlo estimates," Mathematics and Computers in Simulation, vol. 55, no. 1-3, pp. 271-280, 2001.

[48] H. Liu and W. Chen, "Probabilistic Sensitivity Analysis Methods for Design under Uncertainty, Tech B224," Tech. Rep., Integrated Design Automation Laboratory, Department of Mechanical Engineering, Northwestern University.

[49] A. Saltelli and I. M. Sobol', "About the use of rank transformation in sensitivity analysis of model output," Reliability Engineering \& System Safety, vol. 50, no. 3, pp. 225-239, 1995.
[50] T. Homma and A. Saltelli, "Importance measures in global sensitivity analysis of nonlinear models," Reliability Engineering \& System Safety, vol. 52, no. 1, pp. 1-17, 1996.

[51] B. Sudret, "Global sensitivity analysis using polynomial chaos expansions," Reliability Engineering \& System Safety, vol. 93, no. 7, pp. 964-979, 2008.

[52] http://www.climatetechwiki.org/technology/offshore-wind. 


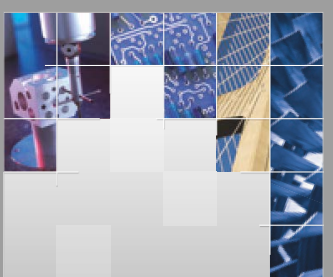

\section{Enfincering}
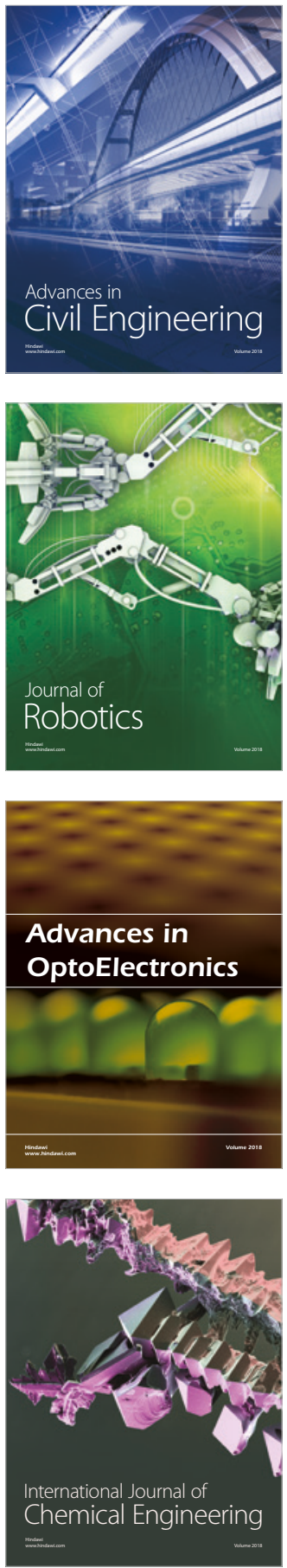

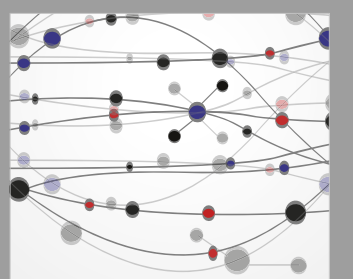

\section{Rotating \\ Machinery}

The Scientific World Journal

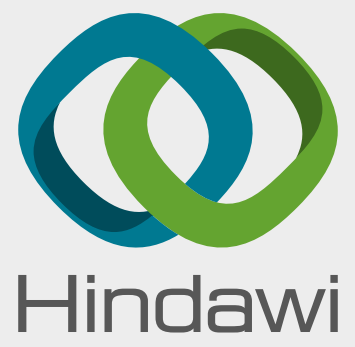

Submit your manuscripts at

www.hindawi.com
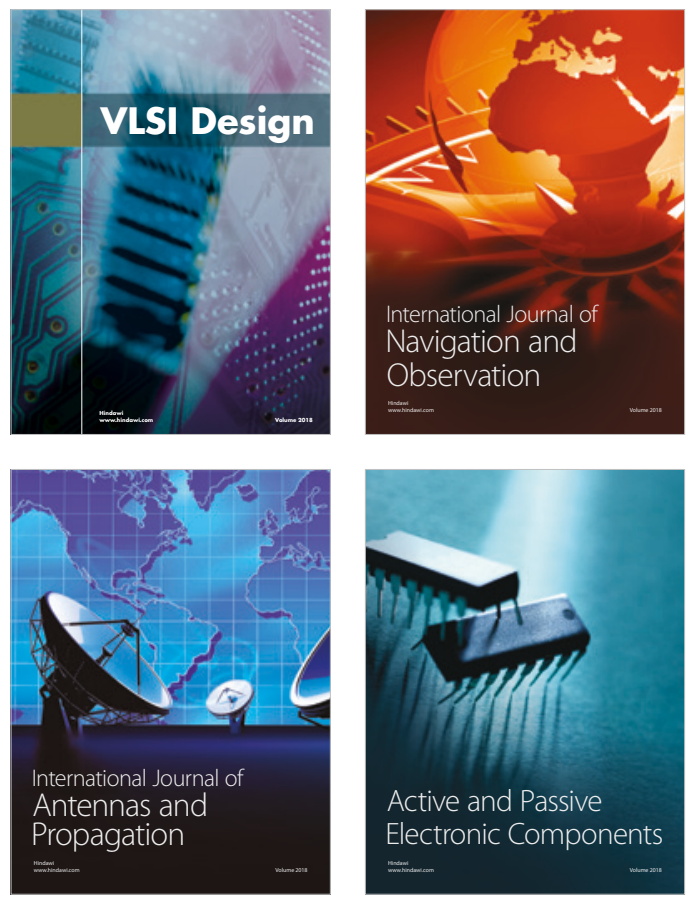
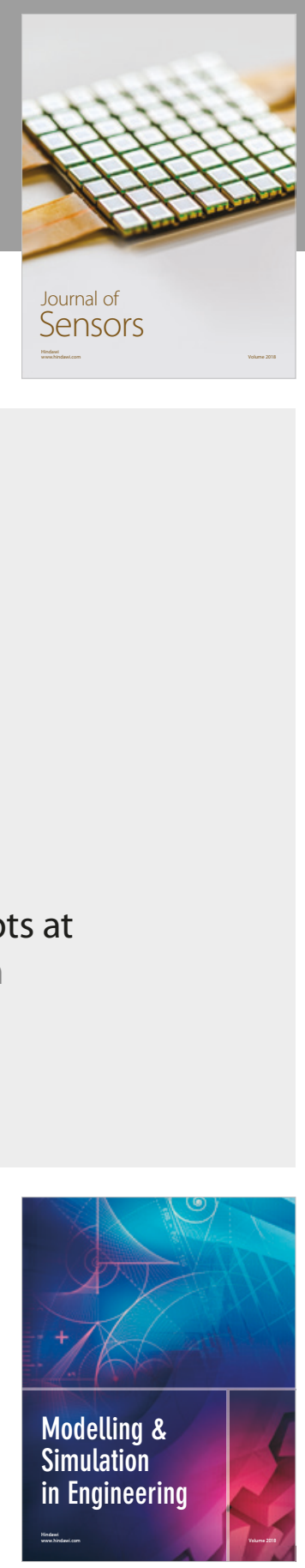

\section{Advances \\ Multimedia}
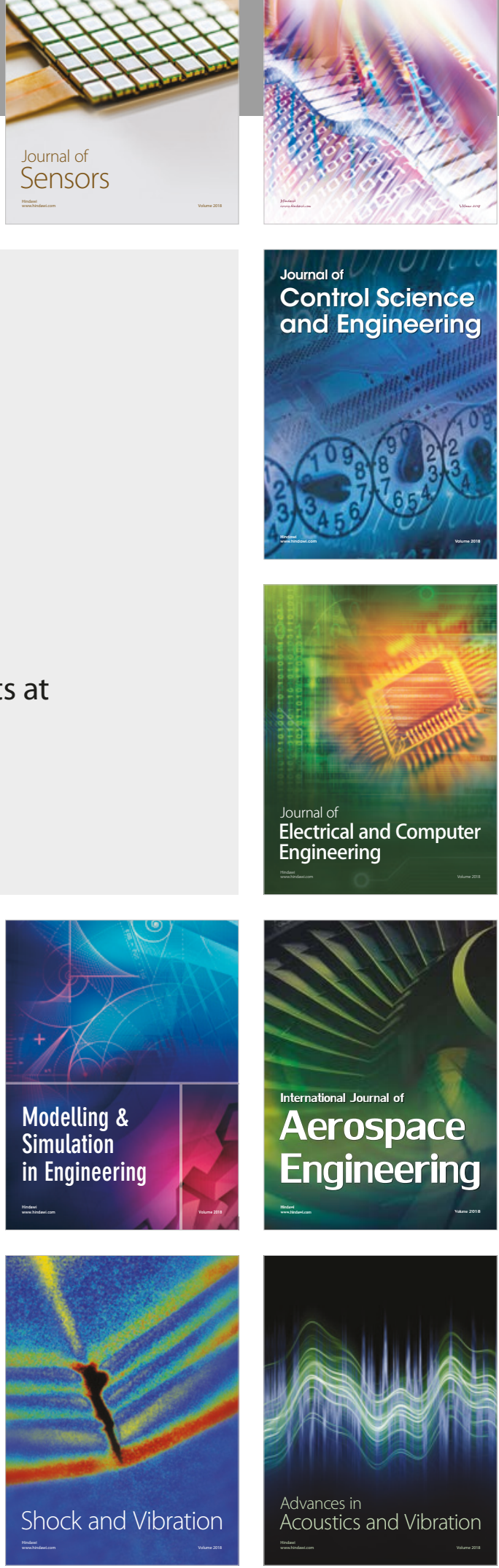